\title{
A characterization of dendroids by the $n$-connectedness of the Whitney levels
}

\author{
by
}

Alejandro Illanes (México, D.F.)

\begin{abstract}
Let $X$ be a continuum. Let $C(X)$ denote the hyperspace of all subcontinua of $X$. In this paper we prove that the following assertions are equivalent: (a) $X$ is a dendroid, (b) each positive Whitney level in $C(X)$ is 2-connected, and (c) each positive Whitney level in $C(X)$ is $\infty$-connected ( $n$-connected for each $n \geq 0$ ).
\end{abstract}

Introduction. Throughout this paper $X$ will denote a continuum (i.e., a compact connected metric space) with metric d. Let $C(X)$ be the hyperspace of all subcontinua of $X$ with the Hausdorff metric $\mathcal{H}$. A Whitney map for $C(X)$ is a continuous function $\mu: C(X) \rightarrow \mathbb{R}$ satisfying: (a) $\mu(\{x\})=0$ for each $x \in X$, (b) if $A, B \in C(X)$ and $A \varsubsetneqq B$, then $\mu(A)<\mu(B)$, and (c) $\mu(X)=1$. A (positive) Whitney level is a set of the form $\mu^{-1}(t)$ where $0 \leq t \leq 1$ (resp. $0<t \leq 1$ ). $S^{n}$ denotes the $n$-sphere. A space $Y$ is $n$ connected if, for every $0 \leq i \leq n$, each map $f: S^{i} \rightarrow Y$ is null homotopic; $Y$ is $\infty$-connected if it is $n$-connected for each $n$. A topological property $\mathrm{P}$ is a Whitney property provided whenever a continuum $X$ has property $\mathrm{P}$, so does every positive Whitney level in $C(X)$. A map is a continuous function. The unit closed interval is denoted by $I$, and the set of positive integers by $\mathbb{N}$.

Positive Whitney levels are continua [1]. Answering questions by J. Krasinkiewicz and S. B. Nadler, Jr., in [9] A. Petrus showed that if $D$ is a 2cell, then there exists a Whitney level $\mathcal{A}$ in $C(D)$ which is not contractible, in fact $\mathcal{A}$ has non-trivial fundamental group and non-trivial first singular homology group.

The main theorem in this paper is:

THEOREM. The following assertions are equivalent:

(i) $X$ is a dendroid,

(ii) Each positive Whitney level in $C(X)$ is 2-connected.

(iii) Each positive Whitney level in $C(X)$ is $\infty$-connected. 
We divide the proof into two independent sections. In the first section we prove that (ii) $\Rightarrow(\mathrm{i})$, and in the second one we prove that (i) $\Rightarrow$ (iii).

1. 2-connectedness of Whitney levels implies that $X$ is a dendroid. We will need the following lemma.

1.1. Lemma. Let $\mu: C(X) \rightarrow \mathbb{R}$ be a Whitney map. Let $t_{0} \in I$. Let $Y$ be a continuum such that $C(Y)$ is contractible. Then every map $f$ : $Y \rightarrow \mu^{-1}\left(\left[0, t_{0}\right]\right)$ is homotopic to a map $g: Y \rightarrow \mu^{-1}\left(\left[0, t_{0}\right]\right)$ such that $\operatorname{Im} g \subset \mu^{-1}\left(t_{0}\right)$.

Proof. Take a map $f: Y \rightarrow \mu^{-1}\left(\left[0, t_{0}\right]\right)$. Since $C(Y)$ is contractible, by [12, Thm. 16.7] there exists a map $F: Y \times I \rightarrow C(Y)$ such that, for every $y \in Y, F(y, 0)=\{y\}, F(y, 1)=Y$ and $s \leq t$ implies that $F(y, s) \subset F(y, t)$.

We distinguish two cases:

(a) $\mu(\bigcup f(Y))=\mu(\bigcup\{f(y) \in C(X): y \in Y\}) \geq t_{0}$. Define $G: Y \times I \rightarrow$ $C(X)$ by $G(y, t)=\bigcup f(F(y, t))=\bigcup\{f(v) \in C(X): v \in F(y, t)\}$ Then $G$ is a map such that $G(y, 0)=f(y)$ and $G(y, 1)=\bigcup f(Y)$ for every $y \in Y$. Define $K: Y \times I \rightarrow \mu^{-1}\left(\left[0, t_{0}\right]\right)$ by

$$
K(y, t)= \begin{cases}G(y, t) & \text { if } \mu(G(y, t)) \leq t_{0}, \\ G(y, s) & \text { if } \mu(G(y, t)) \geq t_{0},\end{cases}
$$

where $s \in\left[0, t_{0}\right]$ is chosen in such a way that $\mu(G(y, s))=t_{0}$.

Then $K(y, 0)=f(y)$ and $K(y, 1) \in \mu^{-1}\left(t_{0}\right)$, and we define $g: Y \rightarrow$ $\mu^{-1}\left(\left[0, t_{0}\right]\right)$ by $g(y)=K(y, 1)$ for every $y \in Y$.

(b) $\mu(\bigcup f(Y)) \leq t_{0}$. Defining $G$ as in (a), we see that $f$ is homotopic (within $\mu^{-1}\left(\left[0, t_{0}\right]\right)$ ) to the constant map $y \rightarrow \bigcup f(Y)$. Since $\bigcup f(Y) \in$ $\mu^{-1}\left(\left[0, t_{0}\right]\right)$, there exists an ordered arc $([12$, Thm. 1.8]) joining $\bigcup f(Y)$ to an element $A_{0} \in \mu^{-1}\left(t_{0}\right)$ (within $\mu^{-1}\left(\left[0, t_{0}\right]\right)$ ). Then we complete the proof of the lemma by defining $g(y)=A_{0}$ for every $y \in Y$.

We will use the following notions related to Whitney levels:

The space of Whitney levels, $N(X)$, of $X$ is defined by $N(X)=\{\mathcal{A} \in$ $C(C(X)): \mathcal{A}$ is a Whitney level in $C(X)\}$. This space was introduced in [5]-[7]. In [7, Lemma 2.2] it was proved that an equivalent metric for $N(X)$ is $\mathcal{H}^{*}(\mathcal{A}, \mathcal{B})=\max \{\mathcal{H}(A, B): A \in \mathcal{A}, B \in \mathcal{B}$ and $A \subset B\}$. A partial order for $N(X)$ is defined in [5] by $\mathcal{A} \leq \mathcal{B}$ if and only if for each $B \in \mathcal{B}$, there exists $A \in \mathcal{A}$ such that $A \subset B$. If $\mathfrak{A} \subset N(X)$ is compact and $\gamma$ is an ordered arc in $C(X)$ beginning with a singleton and ending with $X$, then ([5]) $A_{\gamma}=\bigcap\{A \in \gamma$ : there exists $\mathcal{A} \in \mathfrak{A}$ such that $A \in \mathcal{A}\} \in \gamma \cap \mathcal{B}$ for some $\mathcal{B} \in \mathfrak{A}$. Finally, in [5] it is shown that $\inf (\mathfrak{A})=\left\{\mathfrak{A}_{\gamma} \in C(X): \gamma\right.$ is an ordered arc in $C(X)$ beginning with a singleton and ending with $X\}$ is a Whitney level which is the infimum, in $(N(X), \leq)$, of the set $\mathfrak{A}$. 
Conventions. $\mathbb{R}^{n}$ denotes the Euclidean $n$-dimensional space. $e: \mathbb{R} \rightarrow$ $S^{1}$ denotes the exponential map defined by $\mathrm{e}(t)=(\cos t, \sin t) . D^{2}$ is the unit disk in $\mathbb{R}^{2}$. If $Y$ is a topological space, a map $f: Y \rightarrow S^{1}$ can be lifted $(f \simeq 1)$ if there exists a map $g: Y \rightarrow \mathbb{R}$ such that $\mathrm{e} \circ g=f$ (equivalently, if $f$ is null homotopic, see [10, Lemma 5]). If $A \in C(X)$ and $\varepsilon>0$ then $N(\varepsilon, A)$ denotes the set $\{x \in X$ : there exists $y \in A$ such that $\mathrm{d}(x, y)<\varepsilon\}$ and $B(A, \varepsilon)$ denotes the set $\{B \in C(X): \mathcal{H}(A, B)<\varepsilon\} .2^{X}$ denotes the hyperspace of all closed nonempty connected subsets of $X$.

From now on, in this section, we will suppose that if $\mathcal{A}$ is a positive Whitney level in $C(X)$, then every map $f: S^{i} \rightarrow \mathcal{A}$ is null homotopic for $i=1,2$ (we are not supposing yet that $\mathcal{A}$ is pathwise connected).

\subsection{Theorem. $X$ is hereditarily unicoherent.}

P r o of. Suppose, on the contrary, that there exist $A_{1}, B_{1} \in C(X)$ such that $A_{1} \cap B_{1}$ is not connected. Let $H, K \in 2^{X}$ be such that $H \cap K=\emptyset$ and $A_{1} \cap B_{1}=H \cup K$. We will construct:

(a) A Whitney map $\omega$ for $C(X)$,

(b) A number $t_{0} \in(0,1]$,

(c) Two open subsets $\mathcal{V}_{1}$ and $\mathcal{V}_{2}$ in $\omega^{-1}\left(\left[0, t_{0}\right]\right)$,

(d) A map $\lambda: S^{1} \rightarrow \mathcal{V}_{1} \cap \mathcal{V}_{2}$ and

(e) A map $h_{1}: \mathcal{V}_{1} \cap \mathcal{V}_{2} \rightarrow S^{1}$

such that $\omega^{-1}\left(\left[0, t_{0}\right]\right)=\mathcal{V}_{1} \cup \mathcal{V}_{2}, h_{1} \circ \lambda$ is not homotopic to a constant and, for $i=1,2, \lambda: S^{1} \rightarrow \mathcal{V}_{i}$ can be extended to the disk $D^{2}$. Then, using Lemma 1.1 and a Mayer-Vietoris type sequence we will obtain a contradiction. The construction of these elements is divided into a sequence of steps.

A. There exists $A_{0} \in C(X)$ such that $A_{0} \subset A_{1}, A_{0} \cap H \neq \emptyset, A_{0} \cap K \neq \emptyset$ and $A_{0}$ is minimal with these properties.

To construct $A_{0}$, choose a Whitney map $\mu$ for $C(X)$. Let $t_{1}=\min \{\mu(A)$ $\in I: A \subset A_{1}, A \cap H \neq \emptyset$ and $\left.A \cap K \neq \emptyset\right\}$. Take $A_{0} \in C(X)$ such that $\mu\left(A_{0}\right)=t_{1}$.

B. Let $H_{1}=A_{0} \cap H$ and $K_{1}=A_{0} \cap K$. Then there exists $B_{0} \in C(X)$ such that $B_{0} \subset B_{1}, B_{0} \cap H_{1} \neq \emptyset, B_{0} \cap K_{1} \neq \emptyset$ and $B_{0}$ is minimal with these properties. Define $H_{0}=H_{1} \cap B_{0}$ and $K_{0}=K_{1} \cap B_{0}$. Then $A_{0} \cap B_{0}=H_{0} \cup K_{0}$, $H_{0} \cap K_{0}=\emptyset$ and $H_{0}, K_{0} \in 2^{X}$. Furthermore, if $A$ (resp. $B$ ) is a proper subcontinuum of $A_{0}$ (resp. $B_{0}$ ), then $A \cap H_{0}=\emptyset$ (resp. $B \cap H_{0}=\emptyset$ ) or $A \cap K_{0}=\emptyset$ (resp. $B \cap K_{0}=\emptyset$ ).

C. Let $E=A_{0} \cup B_{0}$. Let $S^{+}=\left\{(x, y) \in S^{1}: y \geq 0\right\}$ and $S^{-}=$ $\left\{(x, y) \in S^{1}: y \leq 0\right\}$. Since $X$ is metric, Tietze's Theorem implies that there exists a map $f_{0}: E \rightarrow S^{1}$ such that $H_{0}=f_{0}^{-1}((-1,0)), K_{0}=f_{0}^{-1}((1,0))$, $f_{0}\left(A_{0}\right) \subset S^{+}$and $f_{0}\left(B_{0}\right) \subset S^{-}$. Since $S^{1}$ is an ANR (metric), there exists 
an open subset $U$ in $X$ and a map $f: U \rightarrow S^{1}$ such that $E \subset U$ and $f \mid E=f_{0}$. Then the Unique Lifting Theorem implies that $f \mid E$ cannot be lifted.

D. If $A$ is a proper subcontinuum of $E$, then $f \mid A \simeq 1$.

To see this, suppose, for example, that $A_{0}$ is not contained in $A$. Let $A_{H}=\bigcup\left\{L \in C(X): L\right.$ is a component of $A \cap A_{0}$ and $\left.L \cap H_{0} \neq \emptyset\right\}$ and let $A_{K}=\bigcup\left\{L \in C(X): L\right.$ is a component of $A \cap A_{0}$ and $\left.L \cap H_{0}=\emptyset\right\}$. Then $A_{H}$ is closed in $X$. We will prove that $A_{K}$ is closed. If $A \subset A_{0}$, then either $A_{K}=A$ or $A_{K}=\emptyset$. Suppose then that $A$ is not contained in $A_{0}$. If $L$ is a component of $A \cap A_{0}$, then ([12, Thm. 20.2]) $L$ intersects either $H_{0}$ or $K_{0}$ but not both of them. If $x \in \mathrm{Cl}\left(A_{K}\right)$ then $x=\lim x_{n}$ where $\left(x_{n}\right)_{n}$ is a sequence such that, for each $n, x_{n} \in L_{n}$ for some component $L_{n}$ of $A_{0} \cap A$ such that $L_{n} \cap H_{0}=\emptyset$ (then $L_{n} \cap K_{0} \neq \emptyset$ ). Therefore the component $L$ of $A_{0} \cap A$ which contains $x$ intersects $K_{0}$. Hence $L \cap H_{0}=\emptyset$ and $x \in A_{K}$. The minimality of $A_{0}$ implies that $A_{H} \cap K_{0}=\emptyset$. Notice that $A_{H} \cap A_{K}=\emptyset$ and $A_{K} \cap H_{0}=\emptyset$.

Thus $A=A_{H} \cup A_{K} \cup\left(A \cap B_{0}\right)$. Since $A_{H}, A_{K} \subset A_{0}=f^{-1}\left(S^{+}\right)$and $A \cap B_{0} \subset B_{0}=f^{-1}\left(S^{-}\right)$, we find that $f\left|A_{H}, f\right| A_{K}$ and $f \mid\left(A \cap B_{0}\right)$ can be lifted. Since $A_{H} \cap A \cap B_{0} \subset H_{0}=f^{-1}((-1,0)), A_{K} \cap A \cap B_{0} \subset K_{0}=$ $f^{-1}((1,0))$ and $A_{H} \cap A_{K}=\emptyset$, it follows that $f \mid A$ can be lifted.

E. There exists an open subset $\mathcal{V}$ of $C(X)$ such that $C(E)-\{E\} \subset \mathcal{V}$ and for each $A \in \mathcal{V}, A \subset U$ and $f \mid A \simeq 1$.

Indeed, let $A \in C(E)-\{E\}, f \mid A \simeq 1$. Then ([2]) there exists an open subset $U_{A}$ of $U$ containing $A$ such that $f \mid U_{A} \simeq 1$. Therefore there exists $\varepsilon_{A}>0$ such that if $\mathcal{H}(A, B)<\varepsilon_{A}$, then $f \mid B \simeq 1$. Define $\mathcal{V}=\{B \in C(X)$ : $\mathcal{H}(A, B)<\varepsilon_{A}$ for some $\left.A \in C(E)-\{E\}\right\}$.

F. Fix a Whitney map $\nu_{0}: 2^{X} \rightarrow I$. Let $\nu=\nu_{0} \mid C(X)$. Define $t^{*}=\nu(E)>0$ and define $h: C(X) \times I \times\left(0, t^{*}\right) \rightarrow \mathbb{R}$ by $h(A, t, s)=$ $\min \left\{\nu(A) t^{*} / s, \nu_{0}(A \cup E)+t(\nu(A)-\nu(E))\right\}$. Then $h$ is continuous and $h(E, t, s)=t^{*}$ for every $t \in I$ and $s \in\left(0, t^{*}\right)$. Fix $t \in(0,1]$ and $s \in\left(0, t^{*}\right)$. Then the map $A \rightarrow h(A, t, s) / h(X, t, s)$ from $C(X)$ to $I$ is a Whitney map.

G. If $0<s_{1}<s_{2}<t^{*}$, then there exists $r \in(0,1]$ such that if $0<t \leq r$, $A \in \nu^{-1}\left(\left[s_{1}, s_{2}\right]\right)$ and $h\left(A, t, s_{1}\right)<t^{*}$, then $A \in \mathcal{V}$.

Indeed, otherwise we can choose sequences $\left(t_{n}\right)_{n} \subset(0,1]$ and $\left(D_{n}\right)_{n} \subset$ $\nu^{-1}\left(\left[s_{1}, s_{2}\right]\right)$ such that $t_{n} \rightarrow 0$ and $h\left(D_{n}, t_{n}, s_{1}\right)<t^{*}$ and $D_{n} \notin \mathcal{V}$ for all $n$. We may suppose that $D_{n} \rightarrow A$ for some $A \in \nu^{-1}\left(\left[s_{1}, s_{2}\right]\right)$. Then $A \notin \mathcal{V}$ and $\nu(A) \leq s_{2}<\nu(E)$. Thus $A$ is not contained in $E$ and $\nu_{0}(A \cup E)>t^{*}$. Since $t_{n}\left(\nu\left(D_{n}\right)-\nu(E)\right)+\nu_{0}\left(D_{n} \cup E\right) \rightarrow \nu_{0}(A \cup E)$ and $\nu\left(D_{n}\right) t^{*} / s_{1} \geq t^{*}$, we conclude that there exists $n \in \mathbb{N}$ such that $h\left(D_{n}, t_{n}, s_{1}\right) \geq t^{*}$. This contradiction completes the proof of $\mathrm{G}$. 
H. Choose a sequence $\left(s_{n}\right)_{n} \subset\left(0, t^{*}\right)$ such that $s_{n} \rightarrow t^{*}$ and $0<s_{1}<$ $s_{2}<\ldots$ Let $\left(t_{n}\right)_{n} \subset(0,1]$ be a sequence such that $t_{n} \rightarrow 0, t_{1}>t_{2}>\ldots$ and, for each $n$, if $A \in \nu^{-1}\left(\left[s_{n}, s_{n+1}\right]\right)$ and $h\left(A, t_{n}, s_{n}\right)<t^{*}$, then $A \in \mathcal{V}$.

I. Let $\mathcal{A}=\nu^{-1}\left(t^{*}\right)$. For each $n$, define $\mathcal{A}_{n}=\left\{A \in C(X): h\left(A, t_{n}, s_{n}\right)=\right.$ $\left.t^{*}\right\}$. Then $E \in \mathcal{A}_{n}, \mathcal{A}_{n}$ is a positive Whitney level, $\nu^{-1}\left(s_{n}\right) \leq \mathcal{A}_{n} \leq \mathcal{A}$ and $\mathcal{A}_{n} \rightarrow \mathcal{A}$.

To see this, let $A \in \mathcal{A}_{n}$; then $t^{*} \leq \nu(A) t^{*} / s_{n}$. Thus $s_{n} \leq \nu(A)$. Then there exists $B \in \nu^{-1}\left(s_{n}\right)$ such that $B \subset A$. Hence $\nu^{-1}\left(s_{n}\right) \leq \mathcal{A}_{n}$. Now, let $A \in \mathcal{A}$. Then $h\left(A, t_{n}, s_{n}\right)=\min \left\{\nu_{0}(A \cup E),\left(t^{*}\right)^{2} / s_{n}\right\}$. Therefore $h\left(A, t_{n}, s_{n}\right) \geq t^{*}$, so that there exists $B \in C(X)$ such that $B \subset A$ and $h\left(B, t_{n}, s_{n}\right)=t^{*}$. Thus $\mathcal{A}_{n} \leq \mathcal{A}$.

By $[7$, Lemma $2.2(\mathrm{~b})], \mathcal{H}^{*}\left(\mathcal{A}_{n}, \mathcal{A}\right) \leq \mathcal{H}^{*}\left(\nu^{-1}\left(s_{n}\right), \nu^{-1}\left(t^{*}\right)\right) \rightarrow 0$. Hence $\mathcal{A}_{n} \rightarrow \mathcal{A}$.

J. Define $\mathcal{B}=\inf \left(\{\mathcal{A}\} \cup\left\{\mathcal{A}_{n}: n \geq 1\right\}\right)$. Then $\mathcal{B}$ is a Whitney level. Thus there exists $t_{0} \in I$ and a Whitney map $\mu$ for $C(X)$ such that $\mathcal{B}=\mu^{-1}\left(t_{0}\right)$. Since $E \in \mathcal{A}$ and $E \in \mathcal{A}_{n}$ for all $n$, it follows that $E \in \mathcal{B}$ and $t_{0}>0$.

$\mathrm{K}$. The set $\mathcal{W}=\nu^{-1}\left(\left(s_{1}, t^{*}\right)\right) \cap \mu^{-1}\left(\left[0, t_{0}\right)\right)$ is contained in $\mathcal{V}$.

Indeed, let $A \in \mathcal{W}$. Then there exists $N$ such that $A \in \nu^{-1}\left(\left[s_{N}, s_{N+1}\right]\right)$. By $\mathrm{H}$, we must show that $h\left(A, t_{N}, s_{N}\right)<t^{*}$. Suppose, on the contrary, that $h\left(A, t_{N}, s_{N}\right) \geq t^{*}$. Then there exists a subcontinuum $A^{*}$ of $A$ such that $h\left(A^{*}, t_{N}, s_{N}\right)=t^{*}$. Choose a point $a \in A^{*}$. Let $\gamma$ be an ordered arc in $C(X)$ joining $\{a\}$ to $X$ such that $A^{*}, A \in \gamma$. Let $A_{2}$ be the unique element in $\gamma \cap \mathcal{B}$. Since $\mu(A)<t_{0}=\mu\left(A_{2}\right)$, we find that $A \varsubsetneqq A_{2}$. Thus $A \varsubsetneqq A_{2}=\bigcap\left\{B \in C(X): B \in \gamma \cap\left(\{\mathcal{A}\} \cap\left\{\mathcal{A}_{n}: n \in \mathbb{N}\right\}\right)\right\} \subset A^{*}$. This contradiction proves that $A \in \mathcal{V}$.

L. Choose a Whitney map $\bar{\mu}: 2^{X} \rightarrow I$ which extends $\mu$ (see $[14$, Cor. 3.3]). Define $\omega: C(X) \rightarrow I$ by $\omega(A)=(\bar{\mu}(A \cup E) \bar{\mu}(A))^{1 / 2}$. Then $\omega$ is a Whitney map such that $\omega(E)=\mu(E)=t_{0}, \omega^{-1}\left(t_{0}\right)-\{E\} \subset \mu^{-1}\left(\left[0, t_{0}\right)\right)$ and $\nu^{-1}\left(\left(s_{1}, 1\right]\right) \cap \omega^{-1}\left(t_{0}\right) \subset \mathcal{V} \cup\{E\}$.

To prove this, let $A \in\left(\nu^{-1}\left(\left(s_{1}, 1\right]\right) \cap \omega^{-1}\left(t_{0}\right)\right)-\{E\}$. By K, to show that $A \in \mathcal{V}$, it is enough to prove that $\nu(A)<t^{*}$. Suppose that $\nu(A) \geq t^{*}$. Then there exists $A^{*} \in \nu^{-1}\left(t^{*}\right)$ such that $A^{*} \subset A$. Since $\mathcal{B} \leq \nu^{-1}\left(t^{*}\right)$, there exists $B \in \mathcal{B}$ such that $B \subset A^{*}$. Since $E$ is not contained in $A$, we have $t_{0}=\omega(A) \geq \omega(B)>\mu(B)=t_{0}$. This contradiction proves that $A \in \mathcal{V}$.

M. There exists $\varepsilon>0$ such that $B(E, \varepsilon) \subset \nu^{-1}\left(\left(s_{1}, 1\right]\right)$ and if $\mathcal{H}(A, E)<$ $\varepsilon, A \subset B$ and $B \in \omega^{-1}\left(t_{0}\right)$, then $B \in \mathcal{V} \cup\{E\}$.

Indeed, let $\varepsilon_{1}>0$ be such that if $\mathcal{H}(E, A)<\varepsilon_{1}$ then $A \in \nu^{-1}\left(\left(s_{1}, 1\right]\right)$. Let $\delta>0$ be such that $A \subset B$ and $|\omega(A)-\omega(B)|<\delta$ imply that $\mathcal{H}(A, B)<$ $\varepsilon_{1} / 2$ (see [12, Lemma 1.28]). Choose $r_{0} \in\left[0, t_{0}\right)$ such that $t_{0}-r_{0}<\delta$. Finally, choose $\varepsilon>0$ such that $\varepsilon<\varepsilon_{1} / 2$ and $\mathcal{H}(A, E)<\varepsilon$ imply that 
$A \in \omega^{-1}\left(\left(r_{0}, 1\right]\right)$.

N. Define $\mathcal{V}_{1}=B(E, \varepsilon) \cap \omega^{-1}\left(\left[0, t_{0}\right]\right)$ and $\mathcal{V}_{2}=\omega^{-1}\left(\left[0, t_{0}\right]\right)-\{E\}$. Then $\mathcal{V}_{1}$ and $\mathcal{V}_{2}$ are open subsets of $\omega^{-1}\left(\left[0, t_{0}\right]\right)$ such that $\omega^{-1}\left(\left[0, t_{0}\right]\right)=\mathcal{V}_{1} \cup \mathcal{V}_{2}$ and if $A \in \mathcal{V}_{1} \cap \mathcal{V}_{2}$, then $f \mid A \simeq 1$.

O. Define $h_{1}: \mathcal{V}_{1} \cap \mathcal{V}_{2} \rightarrow S^{1}$ in the following way: Given $A \in \mathcal{V}_{1} \cap \mathcal{V}_{2}$, take a map $g_{A}: A \rightarrow \mathbb{R}$ such that $e \circ g_{A}=f \mid A$. Define $h_{1}(A)=e\left(\min g_{A}(A)\right)$. Then $h_{1}$ is well defined and continuous.

Indeed, it is easy to prove that $h_{1}$ is well defined. To prove that $h_{1}$ is continuous, take a sequence $\left(D_{n}\right)_{n}$ in $\mathcal{V}_{1} \cap \mathcal{V}_{2}$ such that $D_{n} \rightarrow A \in \mathcal{V}_{1} \cap \mathcal{V}_{2}$. Let $g_{A}: A \rightarrow \mathbb{R}$ be a map such that $\operatorname{eog}_{A}=f \mid A$. Let $U_{1}$ be an open subset of $X$ such that $A \subset U_{1} \subset U$ and $f \mid U_{1} \simeq 1$. Let $g: U_{1} \rightarrow \mathbb{R}$ be a map such that e $\circ g=f \mid U_{1}$. Since $D_{n} \rightarrow A$, there exists $N$ such that $D_{n} \subset U_{1}$ for all $n \geq N$. Then, for all $n \geq N, h_{1}\left(D_{n}\right)=e\left(\min g\left(D_{n}\right)\right) \rightarrow e(\min g(A))=h_{1}(A)$.

P. Choose $\delta>0$ such that $A \subset B$ and $|\omega(A)-\omega(B)|<\delta$ imply that $\mathcal{H}(A, B)<\varepsilon$. Choose $s^{*} \in\left(0, t_{0}\right)$ such that $t_{0}-s^{*}<\delta$ and $\omega\left(A_{0}\right), \omega\left(B_{0}\right)<$ $s^{*}$. Choose $p_{0} \in H_{0}$ and $q_{0} \in K_{0}$. Finally, choose maps $\alpha_{1}, \alpha_{2}, \beta_{1}$ and $\beta_{2}$ from $I$ to $C(X)$ such that $\alpha_{1}(0)=\left\{p_{0}\right\}=\beta_{1}(0), \alpha_{2}(0)=\left\{q_{0}\right\}=$ $\beta_{2}(0), \alpha_{1}(1)=A_{0}=\alpha_{2}(1), \beta_{1}(1)=B_{0}=\beta_{2}(1)$ and, for $i=1,2, s<t$ implies that $\alpha_{i}(s)$ (resp. $\beta_{i}(s)$ ) is properly contained in $\alpha_{i}(t)$ (resp. $\beta_{i}(t)$ ) (see [12, Thm. 1.8]). by

Q. Choose $r_{1} \in I$ such that $\omega\left(B_{0} \cup \alpha_{2}\left(r_{1}\right)\right)=s^{*}$. Define $\gamma:[0,4] \rightarrow C(X)$

$$
\gamma= \begin{cases}\alpha_{2}\left((1-t) r_{1}+t\right) \cup \beta_{2}(w(t)) & \text { if } t \in[0,1], \\ \beta_{2}((2-t)(w(1))) \cup A_{0} \cup \beta_{1}(x(t)) & \text { if } t \in[1,2], \\ \beta_{1}((3-t)(x(2))+t-2) \cup \alpha_{1}(y(t)) & \text { if } t \in[2,3], \\ \alpha_{1}((4-t) y(3)) \cup B_{0} \cup \alpha_{2}(z(t)) & \text { if } t \in[3,4] .\end{cases}
$$

Here $w(t), x(t), y(t), z(t) \in I$, for $t$ in the respective intervals, are consecutively chosen in such a way that $\omega(\gamma(t))=s^{*}$ for all $t \in[0,4]$. Then $\gamma$ is well defined, continuous, $\gamma(0)=\gamma(4)$ and $\gamma(t) \in \omega^{-1}\left(s^{*}\right) \cap C(E) \cap \mathcal{V}_{1} \cap \mathcal{V}_{2}$ for every $t \in[0,4]$.

R. Define $\lambda: S^{1} \rightarrow \omega^{-1}\left(s^{*}\right) \cap \mathcal{V}_{1} \cap \mathcal{V}_{2}$ by $\lambda(\cos t, \sin t)=\gamma(2(t+\pi) / \pi)$ if $t \in[-\pi, \pi]$. Then $\lambda$ is well defined, continuous and $h_{1} \circ \lambda$ is not homotopic to a constant.

To see that $h_{1} \circ \lambda$ cannot be lifted, we first show that, for each $z \in S^{-}$, there exists a map $g_{z}: \lambda(z) \rightarrow[-\pi, 2 \pi)$ such that $\mathrm{e} \circ g_{z}=f \mid \lambda(z)$ and $0 \in \operatorname{Im} g_{z}$. Set $z=(\cos t, \sin t)$ with $t \in[-\pi, 0]$. If $t \in[-\pi,-\pi / 2]$, then $s=2(t+\pi) / \pi \in[0,1]$ and $\lambda(z)=\gamma(s)=\alpha_{2}\left((1-s) r_{1}+s\right) \cup \beta_{2}(w(s))$. If $\beta_{2}(w(s))=B_{0}$, then $\alpha_{2}\left((1-s) r_{1}+s\right)$ is a proper subset of $A_{0}$ since $s^{*}<t_{0}$. The minimality of $A_{0}$ implies that $\alpha_{2}\left((1-s) r_{1}+s\right) \cap H_{0}=\emptyset$. Thus $f\left(\alpha_{2}\left((1-s) r_{1}+s\right)\right)$ is a compact subset of $S^{+}-\{(-1,0)\}$ and, since 
$f\left(\beta_{2}(w(s))\right)$ is contained in $S^{-}$, there exists a map $g_{z}: \lambda(z) \rightarrow[-\pi, \pi)$ such that $f \mid \lambda(z)=e \circ g_{z}$. Since $(1,0)=f\left(q_{0}\right) \in f(\lambda(z))$, we have $0 \in \operatorname{Im} g_{z}$. If $\beta_{2}(w(s))$ is a proper subset of $B_{0}$, the minimality of $B_{0}$ implies that $\beta_{2}(w(s)) \cap H_{0}=\emptyset$, so that $f\left(\beta_{2}(w(s))\right)$ is a compact subset of $S^{-}-\{(-1,0)\}$. Thus there exists a map $g_{z}: \lambda(z) \rightarrow(-\pi, \pi]$ such that $\mathrm{e} \circ g_{z}=f \mid \lambda(z)$. In the case that $t \in[-\pi / 2,0]$, similar considerations lead to the existence of $g_{z}$.

Similarly, for each $z \in S^{+}$, there exists a map $g_{z}: \lambda(z) \rightarrow[0,3 \pi)$ such that $\mathrm{e} \circ g_{z}=f \mid \lambda(z)$ and $\pi \in \operatorname{Im} g_{z}$.

If $z \in S^{-}$, then $h_{1}(\lambda(z))=e\left(\min g_{z}(\lambda(z))\right) \in e([-\pi, 0])=S^{-}$, so $h_{1}(\lambda(z)) \in S^{-}$for each $z \in S^{-}$. Since $\lambda((-1,0))=\gamma(0)=\alpha_{2}\left(r_{1}\right) \cup$ $\beta_{2}(w(0))=\alpha_{2}\left(r_{1}\right) \cup B_{0}$ and $f\left(p_{0}\right)=(-1,0)$, it follows that $-\pi$ is in the image of the map $g_{(-1,0)}: \lambda((-1,0)) \rightarrow[-\pi, \pi)$. Then $h_{1}(\lambda((-1,0)))=$ $e(-\pi)=(-1,0)$. Similarly $h_{1}(\lambda((1,0)))=(1,0)$.

Thus $h_{1} \circ \lambda$ is a map from $S^{1}$ to $S^{1}$ sending $S^{+}$into $S^{+}, S^{-}$into $S^{-},(-1,0)$ into $(-1,0)$ and $(1,0)$ into $(1,0)$. This implies that $h_{1} \circ \lambda$ cannot be lifted.

S. $\lambda: S^{1} \rightarrow \mathcal{V}_{1}$ can be extended to a map $\bar{\lambda}: D^{2} \rightarrow \mathcal{V}_{1}$.

To see this, let $F: S^{1} \times I \rightarrow C\left(S^{1}\right)\left(=D^{2}\right)$ be a map such that, for each $x \in S^{1}, F(x, 0)=\{x\}, F(x, 1)=S^{1}$ and $s \leq t$ implies that $F(x, s) \subset F(x, t)$. Define $\bar{\lambda}: S^{1} \times I \rightarrow C(X)$ by $\bar{\lambda}(x, s)=\bigcup\{\lambda(z) \in$ $C(X): z \in F(x, s)\}$. Then $\bar{\lambda}$ is continuous, $\bar{\lambda}(x, 0)=\lambda(x)$ and $\bar{\lambda}(x, 1)=$ $\bigcup\left\{\lambda(z) \in C(X): z \in S^{1}\right\}=E$ for all $x \in S^{1}$. Identifying $D^{2}$ with $\left(S^{1} \times I\right) /\left(S^{1} \times\{1\}\right)$, we deduce that $\bar{\lambda}$ is an extension of $\lambda$ to $D^{2}$. If $x \in S^{1}$ and $s \in I, \lambda(x)=\bar{\lambda}(x, 0) \subset \bar{\lambda}(x, s) \subset E$, then $\mathcal{H}(\bar{\lambda}(x, s), E) \leq \mathcal{H}(\lambda(x), E)<$ $\varepsilon$ and so $\bar{\lambda}(x, s) \in \omega^{-1}\left(\left[0, t_{0}\right]\right)$. Thus $\bar{\lambda}(x, s) \in \mathcal{V}_{1}$ for every $x \in S^{1}$ and $s \in I$.

T. $\lambda: S^{1} \rightarrow \mathcal{V}_{2}$ can be extended to a map $\lambda^{\prime}: D^{2} \rightarrow \mathcal{V}_{2}$.

This follows from the fact that $\operatorname{Im} \lambda \subset \omega^{-1}\left(s^{*}\right) \subset \mathcal{V}_{2}$ and every map from $S^{1}$ into $\omega^{-1}\left(t_{1}\right)$ is homotopic to a constant.

This completes the construction of $\omega, t_{0}, \mathcal{V}_{1}, \mathcal{V}_{2}, \lambda$ and $h_{1}$. Now we consider the Mayer-Vietoris sequences for the triads $\left(V_{1} \cup V_{2}, V_{1}, V_{2}\right)$ and $\left(S^{2}\right.$, $\left.S_{+}^{2}, S_{-}^{2}\right)$ where $S_{+}^{2}=\left\{(x, y, z) \in S^{2}: z \geq 0\right\}$ and $S_{-}^{2}=\left\{(x, y, z) \in S^{2}: z \leq\right.$ $0\}$. Consider the diagram

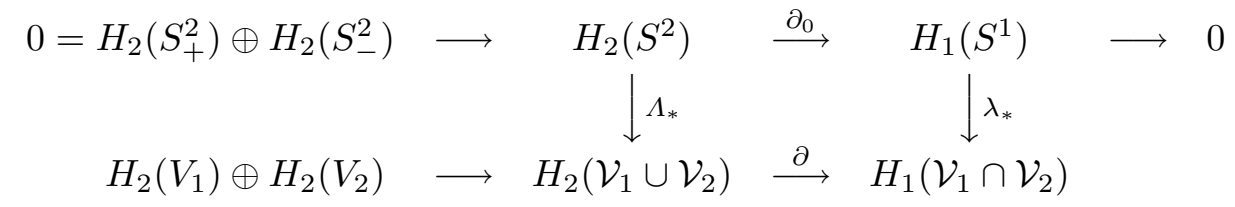

where $\Lambda: S^{2} \rightarrow \mathcal{V}_{1} \cup \mathcal{V}_{2}=\omega^{-1}\left(\left[0, t_{0}\right]\right)$ is defined in such a way that $\Lambda \mid S^{1}=$ $\lambda, \Lambda \mid S_{+}^{2}=\bar{\lambda}$ and $\Lambda \mid S_{-}^{2}=\lambda^{\prime}$. 
By Lemma 1.1, $\Lambda$ is homotopic to a map $\Lambda_{0}: S^{2} \rightarrow \omega^{-1}\left(\left[0, t_{0}\right]\right)$ such that $\operatorname{Im} \Lambda_{0} \subset \omega^{-1}\left(t_{0}\right)$. Since $\omega^{-1}\left(t_{0}\right)$ is a positive Whitney level, $\Lambda_{0}$ is homotopic to a constant. Therefore $\Lambda_{*}$ is the zero homomorphism. This implies that so is $\lambda_{*}$, and hence also the composition $h_{1 *} \circ \lambda_{*}=\left(h_{1} \circ \lambda\right)_{*}$. This is a contradiction since $h_{1} \circ \lambda: S^{1} \rightarrow S^{1}$ is not homotopic to a constant. Therefore $X$ is hereditarily unicoherent.

Remark. If $Y$ is a hereditarily indecomposable continuum then every Whitney level $\mathcal{A}$ in $C(Y)$ is hereditarily indecomposable (see [12, Thm. 14.1]); thus every map from $S^{n}$ into $\mathcal{A}$ is constant for each $n \in \mathbb{N}$. Therefore it is not enough to suppose that the maps from $n$-spheres $(n \geq 1)$ into positive Whitney levels in $C(X)$ are null homotopic to conclude that $X$ is a dendroid. On the other hand [11, Example 3], it is not enough to suppose that every positive Whitney level $\mathcal{A}$ in $C(Z)$ is pathwise connected to conclude that $Z$ is pathwise connected. However, as shown below, it suffices to add the assumption that $Z$ is hereditarily unicoherent.

1.3. LEMMA. Suppose that $Z$ is a hereditarily unicoherent continuum with the following property: If $p, q \in Z$ and $\varepsilon>0$, then there exist $n \in \mathbb{N}$ and $A_{1}, \ldots, A_{n} \in C(Z)$ such that $p \in A_{1}, q \in A_{n}, A_{1} \cap A_{2} \neq \emptyset, \ldots, A_{n-1} \cap A_{n} \neq$ $\emptyset$ and $\operatorname{diam}\left(A_{i}\right)<\varepsilon$ for each $i$. Then $Z$ is pathwise connected.

Proof. Let $p$ and $q$ be two different points in $Z$ and let $A=\bigcap\{B \in$ $C(Z): p, q \in B\}$. Since $Z$ is hereditarily unicoherent, we have $A \in C(Z)$. We will prove that $A$ is connected $i m$ kleinen at each point. Let $a \in A$ and let $\varepsilon>0$. Take $A_{1}, \ldots, A_{n} \in C(Z)$ such that $p \in A_{1}, q \in A_{n}, A_{1} \cap A_{2} \neq$ $\emptyset, \ldots, A_{n-1} \cap A_{n} \neq \emptyset$ and $\operatorname{diam}\left(A_{i}\right)<\varepsilon$ for each $i$. Let $D=\bigcup\left\{A_{i}: a \in A_{i}\right\}$ and let $W=A-\bigcup\left\{A_{i}: a \notin A_{i}\right\}$. Then $D \in C(Z), A \subset A_{1} \cup \ldots \cup A_{n}, W$ is an open subset of $A$ and $a \in W \subset D \subset B(\{a\}, \varepsilon)$. Hence $A$ is connected im kleinen at $a$. Therefore $A$ is a locally connected continuum. Thus $A$ is pathwise connected (in fact, this implies that $A$ is an $\operatorname{arc}$ ). Hence $Z$ is pathwise connected.

1.4. TheOREM. If $Z$ is hereditarily unicoherent and all its positive Whitney levels are pathwise connected, then $Z$ is pathwise connected.

Proof. Let $p, q \in Z$ and let $\varepsilon>0$. Fix a Whitney map $\mu$ for $C(Z)$. Let $0<\delta<1$ be such that if $A, B \in C(Z),|\mu(A)-\mu(B)|<\delta$ and $A \subset B$, then $\mathcal{H}(A, B)<\varepsilon$. Let $0<t \leq \delta / 2$. Choose $A, B \in \mu^{-1}(t)$ such that $p \in A$ and $q \in B$. Let $\alpha: I \rightarrow \mu^{-1}(t)$ be a map such that $\alpha(0)=A$ and $\alpha(1)=B$. Let $\lambda>0$ be such that $|t-s|<\lambda$ implies that $\mathcal{H}(\alpha(t), \alpha(s))<\varepsilon / 3$. Let $0=t_{0}<t_{1}<\ldots<t_{n}=1$ be a partition of $I$ such that $t_{i}-t_{i-1}<\delta$ for all $i \geq 1$. For $i \geq 1$, define $A_{i}=\bigcup\left\{\alpha(t): t_{i-1} \leq t \leq t_{i}\right\}$. Then $A_{1}, \ldots, A_{n} \in C(Z), \operatorname{diam}\left(A_{i}\right)<\varepsilon$ for all $i, p \in A_{1}, q \in A_{n}$ and $A_{1} \cap A_{2} \neq$ $\emptyset, \ldots, A_{n-1} \cap A_{n} \neq \emptyset$. Therefore $Z$ is pathwise connected. 
1.5. TheOREM. If each positive Whitney level in $C(X)$ is 2-connected, then $X$ is a dendroid.

1.6. Corollary. If every positive Whitney level in $C(X)$ is contractibile, then $X$ is a dendroid.

2. If $X$ is a dendroid then every positive Whitney level in $C(X)$ is $\infty$-connected. In [12, Thm. 14.8], it was shown that if $X$ is pathwise connected then every Whitney level for $C(X)$ is also pathwise connected. So we concentrate our attention on the null homotopy of maps from $n$-spheres $(n \geq 1)$ into positive Whitney levels.

Throughout this section we will suppose that $X$ is a dendroid. Fix a Whitney map $\mu$, a number $t_{0} \in(0,1]$ and an integer $N \in \mathbb{N}$. We will show that every map $G: S^{N} \rightarrow \mu^{-1}\left(t_{0}\right)$ is null homotopic. To do this, we will need to define a strong form of convergence in $C(X)$.

2.1. Definition. Given $x \neq y \in X$, the unique arc joining $x$ and $y$ in $X$ will be denoted by $\overline{x y}$. The set $\{x\}$ will be denoted by $\overline{x x}$. Define $L: C(X) \times X \rightarrow C(X)$ by $L(A, x)=\overline{a x}$ where $a$ is the unique element in $A$ such that $\overline{a x} \cap A=\{a\}$. Given a sequence $\left(A_{n}\right)_{n}$ in $C(X)$ and an element $A \in C(X)$, we say that $\left(A_{n}\right)_{n}$ strongly converges to $A\left(A_{n} \stackrel{\mathrm{s}}{\rightarrow} A\right)$ if $A_{n} \rightarrow A$ and $L\left(A_{n}, a\right) \rightarrow\{a\}$ for each $a \in A$.

The following lemma is easy to prove.

2.2. LEMmA. (a) If $A_{n} \stackrel{\mathrm{s}}{\rightarrow} A, B_{n} \stackrel{\mathrm{s}}{\rightarrow} B$ and $A_{n} \cap B_{n} \neq \emptyset$ for each $n$, then $A_{n} \cup B_{n} \stackrel{\mathrm{s}}{\rightarrow} A \cup B$.

(b) Let $\left(A_{n}\right)_{n} \subset C(X)$ and $A \in C(X)$ be such that, for each infinite subset $S$ of $\mathbb{N}$, there exists a subsequence $\left(A_{n_{k}}\right)_{k}$ such that $n_{k} \in S$ for every $k$ and $A_{n_{k}} \stackrel{\mathrm{s}}{\rightarrow} A$. Then $A_{n} \stackrel{\mathrm{s}}{\rightarrow} A$.

Define $J: C(X) \times C(X) \rightarrow C(X)$ by

$$
J(A, B)= \begin{cases}A \cap B & \text { if } A \cap B \neq \emptyset, \\ \{b\} & \text { if } A \cap B=\emptyset,\end{cases}
$$

where $b$ is the unique point in $B$ such that $\overline{a b} \cap B=\{b\}$ for each $a \in A$.

2.3. LEMmA. If $A_{n} \stackrel{\mathrm{s}}{\rightarrow} A$ and $B_{n} \stackrel{\mathrm{s}}{\rightarrow} B$, then $J\left(A_{n}, B_{n}\right) \stackrel{\mathrm{s}}{\rightarrow} J(A, B)$.

Proof. Case 1: $A \cap B=\emptyset$. Then there exists $M$ such that $A_{n} \cap B_{n}=\emptyset$ for all $n \geq M$. Let $\{a\}=J(B, A)$ and $\{b\}=J(A, B)$. For each $n \geq M$, let $\left\{a_{n}\right\}=J\left(B_{n}, A_{n}\right),\left\{b_{n}\right\}=J\left(A_{n}, B_{n}\right)$ and let $c_{n} \in A_{n}$ and $d_{n} \in B_{n}$ be such that $\overline{a c_{n}}=L\left(A_{n}, a\right)$ and $\overline{b d_{n}}=L\left(B_{n}, b\right)$. Since the set $\overline{c_{n} a} \cup \overline{a b} \cup \overline{b d_{n}}$ is connected and intersects $A_{n}$ and $B_{n}$, it contains $\overline{a_{n} b_{n}}$. In particular, $b_{n} \in \overline{c_{n} a} \cup \overline{a b} \cup \overline{b d_{n}} \rightarrow \overline{a b}$. Thus the limit points of the sequence $\left(b_{n}\right)_{n}$ are in $\overline{a b} \cap B=\{b\}$. Therefore $b_{n} \rightarrow b$. Hence $J\left(A_{n}, B_{n}\right) \rightarrow J(A, B)$. 
Since $\overline{c_{n} a} \rightarrow\{a\}$, there exists $M_{1} \geq M$ such that $b_{n} \notin \overline{c_{n} a}$ for every $n \geq$ $M_{1}$. Thus $b_{n} \in \overline{a b} \cup \overline{b d_{n}}$ for all $n \geq M_{1}$. It follows that $\overline{b_{n} b} \rightarrow\{b\}$. So $L\left(J\left(A_{n}, B_{n}\right), b\right) \rightarrow\{b\}$. Thus $J\left(A_{n}, B_{n}\right) \stackrel{\mathrm{s}}{\rightarrow} J(A, B)$.

Case 2: $A \cap B \neq \emptyset$. First we will prove that $\limsup J\left(A_{n}, B_{n}\right) \subset$ $J(A, B)$. Let $x \in \limsup J\left(A_{n}, B_{n}\right)$. Then there exists a subsequence $\left(n_{k}\right)_{k}$ of $(n)_{n}$ and, for each $k$, there exists $x_{k} \in J\left(A_{n_{k}}, B_{n_{k}}\right)$ such that $x_{k} \rightarrow x$. If $A_{n_{k}} \cap B_{n_{k}} \neq \emptyset$ for an infinite number of $k$ 's, then $x \in A \cap B=J(A, B)$ (in this case). Thus we may suppose that $A_{n_{k}} \cap B_{n_{k}}=\emptyset$ for every $k$.

If there exist $z, y \in A \cap B$ such that $z \neq y$, choose $p \in \overline{z y}-\{z, y\}$. For each $k \in \mathbb{N}$, let $a_{k}, c_{k} \in A_{n_{k}}$ be such that $L\left(A_{n_{k}}, z\right)=\overline{a_{k} z}$ and $L\left(A_{n_{k}}, y\right)=\overline{c_{k} y}$. Since $\overline{a_{k} z} \rightarrow\{z\}$ and $\overline{c_{k} y} \rightarrow\{y\}$, there exists $K \in \mathbb{N}$ such that, for all $k \geq K, \overline{a_{k} z} \cap \overline{c_{k} y}=\emptyset, \overline{a_{k} z} \cap \overline{p y}=\emptyset$ and $\overline{p z} \cap \overline{c_{k} y}=\emptyset$. Given $k \geq K$, $\overline{a_{k} c_{k}} \subset A_{n_{k}} \cap\left(\overline{a_{k} z} \cup \overline{z p} \cup \overline{p y} \cup \overline{y c_{k}}\right)$ and $\left(\overline{a_{k} z} \cup \overline{z p}\right) \cap\left(\overline{p y} \cap \overline{y c_{k}}\right)=\{p\}$. Therefore $p \in \overline{a_{k} c_{k}}$. Hence $p \in A_{n_{k}}$ for all $k \geq K$. Similarly, there exists $K_{1}$ such that $p \in B_{n_{k}}$ for all $k \geq K_{1}$. This contradicts our assumption. Therefore $A \cap B$ consists of a single point $a_{0}$.

For each $k \in \mathbb{N}$, let $a_{k} \in A_{n_{k}}$ and $b_{k} \in B_{n_{k}}$ be such that $\overline{a_{k} b_{k}} \cap A_{n_{k}}=$ $\left\{a_{k}\right\}$ and $\overline{a_{k} b_{k}} \cap B_{n_{k}}=\left\{b_{k}\right\}$. Then $\left\{b_{k}\right\}=J\left(A_{n_{k}}, B_{n_{k}}\right)$. So $x_{k}=b_{k}$. Suppose that $L\left(A_{n_{k}}, a_{0}\right)=\overline{c_{k} a_{0}}$ and $L\left(B_{n_{k}}, a_{0}\right)=\overline{d_{k} a_{0}}$ with $c_{k} \in A_{n_{k}}$ and $d_{k} \in B_{n_{k}}$. Then $x_{k} \in \overline{a_{k} b_{k}} \subset \overline{c_{k} a_{0}} \cup \overline{a_{0} d_{k}} \rightarrow\left\{a_{0}\right\}$. Therefore $x=a_{0} \in$ $A \cap B=J(A, B)$. Hence $\limsup J\left(A_{n}, B_{n}\right) \subset J(A, B)$.

Now take a point $x \in J(A, B)=A \cap B$. For each $n$, let $a_{n} \in A_{n}$ and $b_{n} \in \underline{B_{n}}$ be such that $L\left(A_{n}, x\right)=\overline{a_{n} x}$ and $L\left(B_{n}, x\right)=\overline{b_{n} x}$. If $A_{n} \cap B_{n} \neq \emptyset$, then $\overline{a_{n} b_{n}} \subset A_{n} \cup B_{n}$. Thus $\overline{a_{n} b_{n}} \cap A_{n} \cap B_{n} \neq \emptyset$. Hence $\left(\overline{a_{n} x} \cup \overline{x b_{n}}\right) \cap A_{n} \cap$ $B_{n} \neq \emptyset$. This implies that $L\left(A_{n} \cap B_{n}, x\right) \subset \overline{a_{n} x} \cup \overline{x b_{n}}$. If $A_{n} \cap B_{n}=\emptyset$, let $\left\{d_{n}\right\}=J\left(A_{n}, B_{n}\right)$. Then $d_{n} \in \overline{a_{n} x} \cup \overline{x b_{n}}$ and $L\left(J\left(A_{n}, B_{n}\right), x\right) \subset \overline{a_{n} x} \cup \overline{x b_{n}}$. Therefore $L\left(J\left(A_{n}, B_{n}\right), x\right) \subset \overline{a_{n} x} \cup \overline{x b_{n}}$ for all $n$. Since $\overline{a_{n} x} \cup \overline{x b_{n}} \rightarrow\{x\}$, we have $L\left(J\left(A_{n}, B_{n}\right), x\right) \rightarrow\{x\}$. Thus $x \in \liminf J\left(A_{n}, B_{n}\right)$ and we conclude that $J\left(A_{n}, B_{n}\right) \stackrel{\mathrm{s}}{\rightarrow} J(A, B)$.

In order to give a "uniform" parametrization of the $\operatorname{arcs}$ in $X$, we define, for $a, b \in X$, the function $\gamma(a, b): I \rightarrow \overline{a b}$ by $\gamma(a, b)(t)=x$ if $\mu(\overline{a x})=t \mu(\overline{a b})$ and $x \in \overline{a b}$. Then we have:

2.4. Lemma. For each $a, b \in X, \gamma(a, b)$ is a map, $\gamma(a, b)(0)=a, \gamma(a, b)(1)$ $=b$ and, if $a \neq b$, then $\gamma(a, b)$ is injective.

2.5. Lemma. If $\left\{a_{n}\right\} \stackrel{\mathrm{s}}{\rightarrow}\{a\},\left\{b_{n}\right\} \stackrel{\mathrm{s}}{\rightarrow}\{b\}, r_{n} \rightarrow r$ and $t_{n} \rightarrow t$, then $\overline{\gamma\left(a_{n}, b_{n}\right)\left(r_{n}\right) \gamma\left(a_{n}, b_{n}\right)\left(t_{n}\right)} \stackrel{\mathrm{s}}{\rightarrow} \overline{\gamma(a, b)(r) \gamma(a, b)(t)}$ and $\left\{\gamma\left(a_{n}, b_{n}\right)\left(r_{n}\right)\right\} \stackrel{\mathrm{s}}{\rightarrow}$ $\{\gamma(a, b)(r)\}$.

Proof. Let $\gamma_{n}=\gamma\left(a_{n}, b_{n}\right)$ and $\gamma=\gamma(a, b)$. Since $\overline{a_{n} b_{n}} \subset \overline{a_{n} a} \cup \overline{a b} \cup \overline{b b_{n}}$ and $\overline{a b} \subset \overline{a a_{n}} \cup \overline{a_{n} b_{n}} \cup \overline{b_{n} b}$, we have $\overline{a_{n} b_{n}} \rightarrow \overline{a b}$. First, we will show that $\left\{\gamma_{n}\left(r_{n}\right)\right\} \stackrel{\mathrm{s}}{\rightarrow}\{\gamma(r)\}$. 
If $r=0$ or $a=b$, then $\overline{a_{n} \gamma_{n}\left(r_{n}\right)} \rightarrow a$, since $\mu\left(\overline{a_{n} \gamma_{n}\left(r_{n}\right)}\right)=r_{n} \mu\left(\overline{a_{n} b_{n}}\right) \rightarrow$ 0 and $a_{n} \rightarrow a$. Since $L\left(\left\{\gamma_{n}\left(r_{n}\right)\right\}, \gamma(r)\right)=\overline{a \gamma_{n}\left(r_{n}\right)} \subset \overline{a a_{n}} \cup \overline{a_{n} \gamma_{n}\left(r_{n}\right)} \rightarrow\{a\}$, we have $\left\{\gamma_{n}\left(r_{n}\right)\right\} \stackrel{\mathrm{s}}{\rightarrow}\{\gamma(r)\}$.

If $r=1$ and $a \neq b$, then for $p \in \overline{a b}-\{a, b\}, \overline{a_{n} \gamma_{n}\left(r_{n}\right)} \subset \overline{a_{n} a} \cup \overline{a p} \cup \overline{p b} \cup \overline{b b_{n}}$. Since $\mu\left(\overline{a_{n} a} \cup \overline{a p}\right) \rightarrow \mu(\overline{a p})<\mu(\overline{a b})$ and $\mu\left(\overline{a_{n} \gamma_{n}\left(r_{n}\right)}\right)=r_{n} \mu\left(\overline{a_{n} b_{n}}\right) \rightarrow \mu(\overline{a b})$, there exists $M$ such that $\gamma_{n}\left(r_{n}\right) \notin \overline{a_{n} a} \cup \overline{a p}$ for all $n \geq M$. Thus $\gamma_{n}\left(r_{n}\right) \in$ $\overline{p b} \cup \overline{p b_{n}}$ for all $n \geq M$. This implies that $\left\{\gamma_{n}\left(r_{n}\right)\right\} \stackrel{\mathrm{s}}{\rightarrow}\{\gamma(r)\}$.

If $0<r<1$ and $a \neq b$, then for $p \in \overline{a \gamma(r)}-\{\gamma(r)\}$ and $q \in \overline{\gamma(r) b}-\{\gamma(r)\}$, $\overline{a_{n} \gamma_{n}\left(r_{n}\right)} \subset \overline{a_{n} a} \cup \overline{a p} \cup \overline{p q} \cup \overline{q b} \cup \overline{b b_{n}}$. Proceeding as above, there exists $M$ such that $\gamma_{n}\left(r_{n}\right) \notin \overline{a_{n} a} \cup \overline{a p}$ for all $n \geq M$. If there exists a subsequence $\left(\gamma_{n_{k}}\left(r_{n_{k}}\right)\right)_{k}$ of $\left(\gamma_{n}\left(r_{n}\right)\right)_{n}$ such that $\gamma_{n_{k}}\left(r_{n_{k}}\right) \in \overline{q b} \cup \overline{b b_{n_{k}}}$, we may suppose that $\gamma_{n_{k}}\left(r_{n_{k}}\right) \rightarrow x$ for some $x \in \overline{q b}$ and $\overline{a_{n_{k}} \gamma_{n_{k}}\left(r_{n_{k}}\right)} \rightarrow A$ for some $A \in C(X)$. Then $a, x \in A, \mu\left(\overline{a_{n_{k}} \gamma_{n_{k}}\left(r_{n_{k}}\right)}\right) \rightarrow r \mu(\overline{a b})=\mu(\overline{a \gamma(r)})<\mu(\overline{a q}) \leq \mu(\overline{a x}) \leq$ $\mu(A)=\lim \mu\left(\overline{a_{n_{k}} \gamma_{n_{k}}\left(r_{n_{k}}\right)}\right)$. This contradiction proves that there exists $M \in$ $\mathbb{N}$ such that $\gamma_{n}\left(r_{n}\right) \in \overline{p q}$ for all $n \geq M$. It follows that $\left\{\gamma_{n}\left(r_{n}\right)\right\} \stackrel{\mathrm{s}}{\rightarrow}\{\gamma(r)\}$.

Now we will prove that $\overline{\gamma_{n}\left(r_{n}\right) \gamma_{n}\left(t_{n}\right)} \stackrel{\mathrm{s}}{\rightarrow} \overline{\gamma(r) \gamma(t)}$. Notice that $\overline{\gamma_{n}\left(r_{n}\right) \gamma_{n}\left(t_{n}\right)} \rightarrow \overline{\gamma(r) \gamma(t)}$. Given $p=\gamma(s) \in \overline{\gamma(r) \gamma(t)}$, there exists a sequence $\left(s_{n}\right)_{n} \subset I$ such that $s_{n} \rightarrow s$ and $s_{n}$ is between $r_{n}$ and $t_{n}$. Then $\gamma\left(s_{n}\right) \stackrel{\mathrm{s}}{\rightarrow} \gamma(s)$. Since $L\left(\overline{\gamma_{n}\left(r_{n}\right) \gamma_{n}\left(t_{n}\right)}, \gamma(s)\right) \subset \overline{\gamma_{n}\left(s_{n}\right) \gamma_{n}(s)} \rightarrow\{\gamma(s)\}$, we obtain $\overline{\gamma_{n}\left(r_{n}\right) \gamma_{n}\left(t_{n}\right)} \stackrel{\mathrm{s}}{\rightarrow} \overline{\gamma(r) \gamma(t)}$.

Define $\mathfrak{A}=\{(A, B) \in C(X) \times C(X): A \subset B\}$ and $F: \mathfrak{A} \times I \rightarrow C(X)$ by $F(A, B, t)=\bigcup\{\overline{a x} \in C(X): a \in A, x \in B$ and $\mu(\overline{a x}) \leq t\}$.

2.6. LEMMA. (a) $F$ is well defined.

(b) $F \mid\{(A, B)\} \times I$ is continuous for every $(A, B) \in \mathfrak{A}$.

(c) $F(A, B, 0)=A$ and $F(A, B, 1)=B$.

(d) If $s \leq t$, then $F(A, B, s) \subset F(A, B, t)$.

Proof. We only prove (b). Let $(A, B) \in \mathfrak{A}$ and let $\varepsilon>0$. Let $\delta>0$ be such that if $A_{1} \subset B_{1}$ and $\left|\mu\left(A_{1}\right)-\mu\left(B_{1}\right)\right|<\delta$, then $\mathcal{H}\left(A_{1}, B_{1}\right)<\varepsilon$. It is easy to check that if $|s-t|<\delta$, then $\mathcal{H}(F(A, B, t), F(A, B, s))<\varepsilon$. Thus $F \mid\{(A, B)\} \times I$ is continuous.

2.7. Lemma. If $A_{n} \stackrel{\mathrm{s}}{\rightarrow} A, B_{n} \stackrel{\mathrm{s}}{\rightarrow} B$ and $t_{n} \rightarrow t$ with $\left(A_{n}, B_{n}\right) \in \mathfrak{A}$ for each $n$, then $F\left(A_{n}, B_{n}, t_{n}\right) \stackrel{\mathrm{s}}{\rightarrow} F(A, B, t)$.

Proof. Take $x \in \limsup F\left(A_{n}, B_{n}, t_{n}\right)$. Then $x=\lim x_{k}$ where $x_{k} \in$ $F\left(A_{n_{k}}, B_{n_{k}}, t_{n_{k}}\right)$ and $\left(n_{k}\right)_{k}$ is a subsequence of $(n)_{n}$. For each $k$, there exists $a_{k} \in A_{n_{k}}$ and $b_{k} \in B_{n_{k}}$ such that $x_{k} \in \overline{a_{k} b_{k}}$ and $\mu\left(\overline{a_{k} b_{k}}\right) \leq t_{n_{k}}$. We may suppose that $a_{k} \rightarrow a$ for some $a \in A$ and $\overline{a_{k} b_{k}} \rightarrow C$ for some $C \in C(X)$. Then $\overline{a x} \subset C \subset B$ and $\mu(\overline{a x}) \leq \mu(C) \leq t$. Hence $x \in F(A, B, t)$. Therefore $\limsup F\left(A_{n}, B_{n}, t_{n}\right) \subset F(A, B, t)$. 
Now take $x \in F(A, B, t)$. Then $x \in B$ and there exists $a \in A$ such that $\mu(\overline{a x}) \leq t$. Let $s=\mu(\overline{a x})$. Then there exists a sequence $\left(s_{n}\right)_{n}$ with $0 \leq s_{n} \leq t_{n}$ for all $n$ and $s_{n} \rightarrow s$. For each $n \in \mathbb{N}$, let $a_{n} \in A_{n}$ and $x_{n} \in B_{n}$ be such that $L\left(A_{n}, a\right)=\overline{a_{n} a}$ and $L\left(B_{n}, x\right)=\overline{x_{n} x}$. Let $y_{n} \in F\left(A_{n}, B_{n}, t_{n}\right)$ be such that $L\left(F\left(A_{n}, B_{n}, t_{n}\right), x\right)=\overline{y_{n} x}$. If $\mu\left(\overline{a_{n} x_{n}}\right) \leq s_{n}$, define $z_{n}=x_{n}$. If $\mu\left(\overline{a_{n} x_{n}}\right) \geq s_{n}$, let $z_{n}$ be the unique element in $\overline{a_{n} x_{n}}$ such that $\mu\left(\overline{a_{n} z_{n}}\right)=s_{n}$. Then $z_{n} \in F\left(A_{n}, B_{n}, t_{n}\right)$.

If $x=a$, then $L\left(F\left(A_{n}, B_{n}, t_{n}\right), x\right)=\overline{y_{n} a} \subset \overline{a_{n} a} \rightarrow\{a\}$. Therefore $L\left(F\left(A_{n}, B_{n}, t_{n}\right), x\right) \rightarrow\{x\}$. Now suppose that $x \neq a$. Given $p \in \overline{a x}-\{a, x\}$, $z_{n} \in \overline{a_{n} x_{n}} \subset \overline{a_{n} a} \cup \overline{a p} \cup \overline{p x} \cup \overline{x x_{n}}$. Since $\mu\left(\overline{a_{n} a} \cup \overline{a p}\right) \rightarrow \mu(\overline{a p})<s$, there exists $M$ such that $z_{n} \in \overline{p x} \cup \overline{x x_{n}}$ for all $n \geq M$. This implies that $\overline{z_{n} x} \rightarrow$ $\{x\}$. Since $\overline{y_{n} x} \subset \overline{z_{n} x}$, we have $L\left(F\left(A_{n}, B_{n}, t_{n}\right), x\right) \rightarrow\{z\}$. It follows that $F\left(A_{n}, B_{n}, t_{n}\right) \stackrel{\mathrm{s}}{\rightarrow} F(A, B, t)$.

Now we "uniformize" the map $F$. Define $G: \mathfrak{A} \times I \rightarrow C(X)$ by $G(A, B, t)=F(A, B, s)$ where $s$ is chosen in such a way that $\mu(G(A, B, t))=$ $\mu(A)+t(\mu(B)-\mu(A))$.

2.8. Lemma. (a) $G(A, B, 0)=A$ and $G(A, B, 1)=B$.

(b) If $s \leq t$, then $G(A, B, s) \subset G(A, B, t)$.

(c) If $A_{n} \stackrel{\mathrm{s}}{\rightarrow} A, B_{n} \stackrel{\mathrm{s}}{\rightarrow} B$ and $t_{n} \rightarrow t$ with $\left(A_{n}, B_{n}\right) \in \mathfrak{A}$ for each $n$, then $G\left(A_{n}, B_{n}, t_{n}\right) \stackrel{\mathrm{s}}{\rightarrow} G(A, B, t)$.

(d) $G \mid\{(A, B)\} \times I$ is continuous for every $(A, B) \in \mathfrak{A}$.

Proof. We only prove (c). We will use Lemma 2.2(b). Let $S$ be an infinite subset of $\mathbb{N}$. For each $n \in S$, let $G\left(A_{n}, B_{n}, t_{n}\right)=F\left(A_{n}, B_{n}, s_{n}\right)$ with $s_{n} \in I$. Let $G(A, B, t)=F(A, B, s)$. Take a subsequence $\left(n_{k}\right)_{k}$ of $(n)_{n}$ such that $n_{k} \in S$ for all $k$ and $s_{n_{k}} \rightarrow s^{*}$ for some $s^{*} \in I$. Then $G\left(A_{n_{k}}, B_{n_{k}}, t_{n_{k}}\right) \stackrel{\mathrm{s}}{\rightarrow} F\left(A, B, s^{*}\right)$. This yields $\mu\left(F\left(A, B, s^{*}\right)\right)=\lim \left(\mu\left(A_{n_{k}}\right)+\right.$ $\left.t_{n_{k}}\left(\mu\left(B_{n_{k}}\right)-\mu\left(A_{n_{k}}\right)\right)\right)=\mu(G(A, B, t))=\mu(F(A, B, s))$. It follows that $F\left(A, B, s^{*}\right)=F(A, B, s)$. Hence $G\left(A_{n_{k}}, B_{n_{k}}, t_{n_{k}}\right) \stackrel{\mathrm{s}}{\rightarrow} G(A, B, t)$. Therefore $G\left(A_{n}, B_{n}, t_{n}\right) \stackrel{\mathrm{s}}{\rightarrow} G(A, B, t)$.

Now we define "standard" arcs joining elements in $\mu^{-1}\left(t_{0}\right)$. Define $\alpha$ : $\mu^{-1}\left(t_{0}\right) \times \mu^{-1}\left(t_{0}\right) \times I \rightarrow \mu^{-1}\left(t_{0}\right)$ in the following way:

A. If $A \cap B=\emptyset$, let $\{a\}=J(B, A),\{b\}=J(A, B)$ and $\gamma=\gamma(a, b)$.

A.1. If $\mu(\overline{a b}) \leq t_{0}$, let $s_{0}$ be the unique number in $I$ such that $\mu(\overline{a b} \cup$ $\left.G\left(\{a\}, A, s_{0}\right)\right)=t_{0}$ then define

$$
\alpha(A, B, t)= \begin{cases}\overline{a \gamma(3 t)} \cup G(\{a\}, A, s) & \text { if } 0 \leq t \leq 1 / 3, \\ G\left(\{a\}, A,(2-3 t) s_{0}\right) \cup \overline{a b} \cup G(\{b\}, B, s) & \text { if } 1 / 3 \leq t \leq 2 / 3, \\ \overline{\gamma(3 t-2) b} \cup G(\{b\}, B, s) & \text { if } 2 / 3 \leq t \leq 1 .\end{cases}
$$


In the three cases the element $s \in I$ is chosen in such a way that $\mu(\alpha(A, B, t))$ $=t_{0}$.

A.2. If $\mu(\overline{a b}) \geq t_{0}$, let $s_{0}$ and $r_{0}$ be the unique elements in $I$ such that $\mu\left(\overline{a \gamma\left(s_{0}\right)}\right)=t_{0}=\mu\left(\overline{\gamma\left(r_{0}\right) b}\right)$. Then define

$$
\alpha(A, B, t)= \begin{cases}\frac{\overline{a \gamma\left(3 t s_{0}\right)} \cup G(\{a\}, A, s)}{\gamma(s) \gamma\left((2-3 t) s_{0}+3 t-1\right)} & \text { if } 0 \leq t \leq 1 / 3, \\ \frac{\text { where } s \in\left[0,(2-3 t) s_{0}+3 t-1\right]}{\gamma\left(3 t-2+(3-3 t) r_{0}\right) b} \cup G(\{b\}, B, s) & \text { if } 1 / 3 \leq t \leq 2 / 3, \\ \text { if } 2 / 3 \leq t \leq 1,\end{cases}
$$

with $s$ chosen as above.

B. If $A \cap B \neq \emptyset$, define

$$
\alpha(A, B, t)= \begin{cases}A & \text { if } 0 \leq t \leq 1 / 3, \\ G(A \cap B, A, 2-3 t) \cup G(A \cap B, B, s) & \text { if } 1 / 3 \leq t \leq 2 / 3, \\ B & \text { if } 2 / 3 \leq t \leq 1,\end{cases}
$$

with $s$ chosen in the same way.

It is easy to check that $\alpha$ is well defined, $\alpha(A, B, 0)=A$ and $\alpha(A, B, 1)=$ $B$ for all $(A, B) \in \mu^{-1}\left(t_{0}\right) \times \mu^{-1}\left(t_{0}\right)$ and if $A, B \subset A_{0} \in C(X)$, then $\alpha(A, B, t) \subset A_{0}$ for each $t \in I$.

2.9. LEmmA. If $A_{n} \stackrel{\mathrm{s}}{\rightarrow} A, B_{n} \stackrel{\mathrm{s}}{\rightarrow} B$ and $t_{n} \rightarrow t$, then $\alpha\left(A_{n}, B_{n}, t_{n}\right) \stackrel{\mathrm{s}}{\rightarrow}$ $\alpha(A, B, t)\left(A_{n}, B_{n}, A\right.$ and $B$ in $\left.\mu^{-1}\left(t_{0}\right)\right)$.

Proof. We will use Lemma 2.2(b). Let $S$ be an infinite subset of $\mathbb{N}$. We need to analyze several cases.

1. $A \cap B \neq \emptyset$.

1.1. $A_{n_{k}} \cap B_{n_{k}}=\emptyset$ for infinitely many elements $n_{1}<n_{2}<\ldots$ in $S$. For each $k$, let $\left\{a_{n_{k}}\right\}=J\left(B_{n_{k}}, A_{n_{k}}\right)$ and $\left\{b_{n_{k}}\right\}=J\left(A_{n_{k}}, B_{n_{k}}\right)$. Since $\left\{b_{n_{k}}\right\}=J\left(A_{n_{k}}, B_{n_{k}}\right) \stackrel{\mathrm{s}}{\rightarrow} J(A, B)=A \cap B, A \cap B$ consists of a single point $a_{0}$. Then $\left\{a_{n_{k}}\right\}=J\left(B_{n_{k}}, A_{n_{k}}\right) \stackrel{\mathrm{s}}{\rightarrow}\left\{a_{0}\right\}$. For each $k$, let $\gamma_{k}=\gamma\left(a_{n_{k}}, b_{n_{k}}\right)$. It follows that, for all sequences $\left(r_{k}\right)_{k}$ and $\left(m_{k}\right)_{k}$ in $I, \overline{\gamma_{k}\left(r_{k}\right) \gamma_{k}\left(m_{k}\right)} \stackrel{\mathrm{s}}{\rightarrow}\left\{a_{0}\right\}$.

1.1.1. $t_{0}=0$. Then $\mu\left(\overline{a_{n_{k}} b_{n_{k}}}\right) \geq t_{0}$, so $\alpha\left(A_{n_{k}}, B_{n_{k}}, t_{n_{k}}\right)$ is equal to either $\left\{a_{n_{k}}\right\}$, a point in $\overline{\gamma_{k}(0) \gamma_{k}(1)}=\overline{a_{n_{k}} b_{n_{k}}}$ or $\left\{b_{n_{k}}\right\}$. Thus $\alpha\left(A_{n_{k}}, B_{n_{k}}, t_{n_{k}}\right) \stackrel{\mathrm{s}}{\rightarrow}$ $\left\{a_{0}\right\}=A=B=\alpha(A, B, t)$.

1.1.2. $t_{0}>0$. We may suppose that $\mu\left(\overline{a_{n_{k}} b_{n_{k}}}\right)<t_{0}$ for every $k$. For each $k$, let $s_{0}^{k} \in I$ be such that $\mu\left(\overline{a_{n_{k}} b_{n_{k}}} \cup G\left(\left\{a_{n_{k}}\right\}, A_{n_{k}}, s_{0}^{k}\right)\right)=t_{0}$ and let $s_{k}$ be the number chosen so that $\mu\left(\alpha\left(A_{n_{k}}, B_{n_{k}}, t_{n_{k}}\right)\right)=t_{0}$. We may suppose that $s_{k} \rightarrow s^{*}$ for some $s^{*} \in I$ and $s_{0}^{k} \rightarrow s^{\prime}$ for some $s^{\prime} \in I$. Then $\overline{a_{0} a_{0}} \cup G\left(\left\{a_{0}\right\}, A, s^{*}\right)$ is an element of $\mu^{-1}\left(t_{0}\right)$ which is contained in $A$. This implies that $G\left(\left\{a_{0}\right\}, A, s^{*}\right)=A$. But $\mu\left(G\left(\left\{a_{0}\right\}, A, s^{*}\right)\right)=\mu\left(\left\{a_{0}\right\}\right)+$ $s^{*}\left(\mu(A)-\mu\left(\left\{a_{0}\right\}\right)\right)$, and so $s^{*}=1$. We may suppose that one of the following three cases holds: 
1.1.2.1. $t_{n_{k}} \in[0,1 / 3]$ for every $k$. Then $t \in[0,1 / 3]$ and $\alpha\left(A_{n_{k}}, B_{n_{k}}, t_{n_{k}}\right)$ $\stackrel{\mathrm{s}}{\rightarrow} G\left(\left\{a_{0}\right\}, A, s^{\prime}\right)=A=\alpha(A, B, t)$.

1.1.2.2. $t_{n_{k}} \in[1 / 3,2 / 3]$ for every $k$. Then $t \in[1 / 3,2 / 3]$ and we have $\alpha\left(A_{n_{k}}, B_{n_{k}}, t_{n_{k}}\right) \stackrel{\mathrm{s}}{\rightarrow} G\left(\left\{a_{0}\right\}, A,(2-3 t) s^{*}\right) \cup \overline{a_{0} a_{0}} \cup G\left(\left\{a_{0}\right\}, B, s^{\prime}\right)=\alpha(A, B, t)$.

1.1.2.3. $t_{n_{k}} \in[2 / 3,1]$ for every $k$. Then $t \in[2 / 3,1]$ and $\alpha\left(A_{n_{k}}, B_{n_{k}}, t_{n_{k}}\right)$ $\stackrel{\mathrm{s}}{\rightarrow} G\left(\left\{a_{0}\right\}, B, s^{\prime}\right)=B=\alpha(A, B, t)$.

This completes Subcase 1.1.

1.2. $A_{n_{k}} \cap B_{n_{k}} \neq \emptyset$ for infinitely many elements $n_{1}<n_{2}<\ldots$ in $S$. Then we may suppose that one of the following three cases holds:

1.2.1. $t_{n_{k}} \in[0,1 / 3]$ for all $k$. Then $\alpha\left(A_{n_{k}}, B_{n_{k}}, t_{n_{k}}\right)=A_{n_{k}} \stackrel{\mathrm{s}}{\rightarrow} A=$ $\alpha(A, B, t)$.

1.2.2. $t_{n_{k}} \in[1 / 3,2 / 3]$ for all $k$. So $\alpha\left(A_{n_{k}}, B_{n_{k}}, t_{n_{k}}\right)=B_{n_{k}} \stackrel{\mathrm{s}}{\rightarrow} B=$ $\alpha(A, B, t)$.

1.2.3. $t_{n_{k}} \in[2 / 3,1]$ for every $k$. Then $\alpha\left(A_{n_{k}}, B_{n_{k}}, t_{n_{k}}\right)=G\left(A_{n_{k}} \cap\right.$ $\left.B_{n_{k}}, A_{n_{k}}, 2-3 t_{n_{k}}\right) \cup G\left(A_{n_{k}} \cap B_{n_{k}}, B_{n_{k}}, s_{k}\right)$, where $s_{k} \in I$, and we may suppose that $s_{k} \rightarrow s^{\prime}$ for some $s^{\prime} \in I$. Then $\alpha\left(A_{n_{k}}, B_{n_{k}}, t_{n_{k}}\right) \stackrel{\mathrm{s}}{\rightarrow} G(J(A, B)$, $A, 2-3 t) \cup G\left(J(A, B), B, s^{\prime}\right)=\alpha(A, B, t)$.

This completes the proof of Case 1 .

2. $A \cap B=\emptyset$. Then we may suppose that $A_{n} \cap B_{n}=\emptyset$ for every $n \in S$. Here it is necessary to consider the following cases:

2.1. $\mu\left(\overline{a_{n_{k}} b_{n_{k}}}\right) \geq t_{0}$ for infinitely many elements $n_{1}<n_{2}<\ldots$ in $S$.

2.1.1. $t_{n_{k}} \in[0,1 / 3]$ for every $k$.

2.1.2. $t_{n_{k}} \in[1 / 3,2 / 3]$ for every $k$.

2.1.3. $t_{n_{k}} \in[2 / 3,1]$ for every $k$.

2.2. $\mu\left(\overline{a_{n_{k}} b_{n_{k}}}\right) \leq t_{0}$ for infinitely many elements $n_{1}<n_{2}<\ldots$ in $S$.

2.2.1. $t_{n_{k}} \in[0,1 / 3]$ for every $k$.

2.2.2. $t_{n_{k}} \in[1 / 3,2 / 3]$ for every $k$.

2.2.3. $t_{n_{k}} \in[2 / 3,1]$ for every $k$.

All of them can be treated similarly to Case 1 .

Hence, in each one of the cases, infinitely many elements $n_{1}<n_{2}<\ldots$ of $S$ can be obtained such that $\alpha\left(A_{n_{k}}, B_{n_{k}}, t_{n_{k}}\right) \stackrel{\mathrm{s}}{\rightarrow} \alpha(A, B, t)$.

Therefore $\alpha\left(A_{n}, B_{n}, t_{n}\right) \stackrel{\mathrm{s}}{\rightarrow} \alpha(A, B, t)$.

2.10. Construction. For each $r \in \mathbb{N}$, let $S_{r}=(\{0,1\})^{r}$. For each set $E=\left\{A_{\sigma} \in \mu^{-1}\left(t_{0}\right): \sigma \in S_{N}\right\}$ define $f_{E}: I^{N} \rightarrow \mu^{-1}\left(t_{0}\right)$ through the following steps:

$f_{E}\left(a_{1}, \sigma_{1}\right)=\alpha\left(A_{\left(0, \sigma_{1}\right)}, A_{\left(1, \sigma_{1}\right)}, a_{1}\right)$ if $a_{1} \in I$ and $\sigma_{1} \in S_{N-1}$.

$f_{E}\left(a_{1}, a_{2}, \sigma_{2}\right)=\alpha\left(f_{E}\left(a_{1}, 0, \sigma_{2}\right), f_{E}\left(a_{1}, 1, \sigma_{2}\right), a_{2}\right)$ if $a_{1}, a_{2} \in I$ and $\sigma_{2} \in$ $S_{N-2}$. 
If $2 \leq r<N$, then $f_{E}\left(a_{1}, \ldots, a_{r}, \sigma_{r}\right)=\alpha\left(f_{E}\left(a_{1}, \ldots, a_{r-1}, 0, \sigma_{r}\right)\right.$, $\left.f_{E}\left(a_{1}, \ldots, a_{r-1}, 1, \sigma_{r}\right), a_{r}\right)$ for $a_{1}, \ldots, a_{r} \in I$ and $\sigma_{r} \in S_{N-r}$.

If $r=N$, then we set $f_{E}\left(a_{1}, \ldots, a_{N}\right)=\alpha\left(f_{E}\left(a_{1}, \ldots, a_{N-1}, 0\right)\right.$, $\left.f_{E}\left(a_{1}, \ldots, a_{N-1}, 1\right), a_{N}\right)$ for $a_{1}, \ldots, a_{N} \in I$.

The following lemma is easy to prove.

2.11. Lemma. (a) $f_{E}$ is well defined.

(b) If $\left(a_{n}\right)_{n} \subset I^{N}$ and $a \in I^{N}$ are such that $a_{n} \rightarrow$ a then $f_{E}\left(a_{n}\right) \stackrel{\mathrm{s}}{\rightarrow}$ $f_{E}(a)$.

(c) If $A_{\sigma} \subset A \in C(X)$ for each $\sigma \in S_{N}$, then $f_{E}(a) \subset A$ for every $a \in I^{N}$.

2.12. Lemma. Let $p, q \in\{0,1\}$. Let $E=\left\{A_{\sigma}: \sigma \in S_{N}\right\}$ and $D=\left\{B_{\sigma}:\right.$ $\left.\sigma \in S_{N}\right\}$ and let $r \in\{1, \ldots, N\}$ be such that $A_{\left(\sigma_{1}, p, \sigma_{2}\right)}=B_{\left(\sigma_{1}, q, \sigma_{2}\right)}$ for each $\sigma_{1} \in S_{r-1}$ and $\sigma_{2} \in S_{N-r}$. Then $f_{E}\left(a_{1}, p, a_{2}\right)=f_{D}\left(a_{1}, q, a_{2}\right)$ for every $a_{1} \in I^{r-1}$ and $a_{2} \in I^{N-r}$.

Proof. Let $x=\left(x_{1}, \ldots, x_{N}\right), y=\left(y_{1}, \ldots, y_{N}\right) \in I^{N}$ be such that $x_{r}=p, y_{r}=q$ and $x_{i}=y_{i}$ for all $i \neq r$. We will show, by induction on $k$, that if $x_{k+1}, \ldots, x_{N}, y_{k+1}, \ldots, y_{N} \in\{0,1\}$ then $f_{E}(x)=f_{D}(y)$.

Suppose that $k=1$. Let $\sigma=\left(x_{2}, \ldots, x_{N}\right)$ and $\varrho=\left(y_{2}, \ldots, y_{N}\right) \in S_{N-1}$. If $r>1$, then $A_{(0, \sigma)}=B_{(0, \varrho)}, A_{(1, \sigma)}=B_{(1, \varrho)}$ and $x_{1}=y_{1}$. Then $f_{E}(x)=$ $\alpha\left(A_{(0, \sigma)}, A_{(1, \sigma)}, x_{1}\right)=\alpha\left(B_{(0, \varrho)}, B_{(1, \varrho)}, y_{1}\right)=f_{D}(y)$. If $r=1$, then $\sigma=\varrho$. Notice that $f_{E}(x)=A_{(p, \sigma)}$ and $f_{D}(y)=B_{(q, \sigma)}$. Thus $f_{E}(x)=f_{D}(y)$.

Suppose that the assertion holds for $k<n$. Suppose that $x_{k+2}, \ldots, x_{N}$, $y_{k+2}, \ldots, y_{N} \in\{0,1\}$. Then $f_{E}(x)=\alpha\left(f_{E}\left(x_{1}, \ldots, x_{k}, 0, x_{k+2}, \ldots, x_{N}\right)\right.$, $\left.f_{E}\left(x_{1}, \ldots, x_{k}, 1, x_{k+2}, \ldots, x_{N}\right), x_{k+1}\right)=(*)$. If $k+1 \neq r$, the induction hypothesis implies that $(*)=f_{D}(y)$, and if $k+1=r$, then $f_{E}(x)=$ $f_{E}\left(x_{1}, \ldots, x_{k}, p, x_{k+2}, \ldots, x_{N}\right)$, which, by the induction hypothesis, is equal to $f_{D}\left(y_{1}, \ldots, y_{k}, q, y_{k+2}, \ldots, y_{N}\right)=f_{D}(y)$.

This completes the induction. Then the theorem follows by taking $k=N$.

2.13. Construction. Let $g: I^{N} \rightarrow \mu^{-1}\left(t_{0}\right)$ be a map. Given $m \in$ $\mathbb{N} \cup\{0\}$ and $x=\left(x_{1}, \ldots, x_{N}\right) \in\left(\left\{0,1, \ldots, 10^{m}-1\right\}\right)^{N}$, define $Q(x)=$ $\left[x_{1} / 10^{m},\left(x_{1}+1\right) / 10^{m}\right] \times \ldots \times\left[x_{N} / 10^{m},\left(x_{N}+1\right) / 10^{m}\right]$ and $E(x)=\left\{A_{\sigma}:\right.$ $\left.\sigma \in S_{N}\right\}$ where $A_{\sigma}=g\left((x+\sigma) / 10^{m}\right)$ for every $\sigma \in S_{N}$. Next, define $h_{x}: Q(x) \rightarrow \mu^{-1}\left(t_{0}\right)$ by $h_{x}(a)=f_{E(x)}\left(10^{m}\left(a-x / 10^{m}\right)\right)$. Then $h_{x}$ is well defined. Now define $h_{m}: I^{N} \rightarrow \mu^{-1}\left(t_{0}\right)$ by $h_{m}(a)=h_{x}(a)$ if $a \in Q(x)$. Finally, define $h: I^{N+1} \rightarrow \mu^{-1}\left(t_{0}\right)$ by

$$
h(a, t)= \begin{cases}g(a) & \text { if } t=0, \\ \alpha\left(h_{m+1}(a), h_{m}(a), 2^{m+1}\left(t-1 / 2^{m+1}\right)\right) & \text { if } t \in\left[1 / 2^{m+1}, 1 / 2^{m}\right] .\end{cases}
$$


2.14. Lemma. For each $m, h_{m}$ is well defined and, if $a_{n} \rightarrow a$, then $h_{m}\left(a_{n}\right) \stackrel{\mathrm{s}}{\rightarrow} h_{m}(a)$.

Proof. To see that $h_{m}$ is well defined take a point $a \in Q(x) \cap Q(y)$. First suppose that $x$ and $y$ differ just in one coordinate $r$. Suppose that $x_{r}<y_{r}$. Then $a_{r} 10^{m}=y_{r}=x_{r}+1$. Then $h_{m}(a)$ can be defined as $f_{E(x)}\left(10^{m}\left(a-x / 10^{m}\right)\right)$ and $f_{E(y)}\left(10^{m}\left(a-y / 10^{m}\right)\right)$ where $E(x)=\{g((x+$ $\left.\left.\sigma) / 10^{m}\right): \sigma \in S_{N}\right\}$ and $E(y)=\left\{g\left((y+\sigma) / 10^{m}\right): \sigma \in S_{N}\right\}$.

We will apply Lemma 2.12. Let $c=10^{m}\left(a-x / 10^{m}\right)$ and $d=10^{m}(a-$ $\left.y / 10^{m}\right)$. Then $c_{r}=1$ and $d_{r}=0$. Let $p=1$ and $q=0$. For $\sigma_{1} \in S_{r-1}$ and $\sigma_{2} \in S_{N-r}$ we have $g\left(\left(x+\left(\sigma_{1}, p, \sigma_{2}\right)\right) / 10^{m}\right)=g\left(\left(y+\left(\sigma_{1}, q, \sigma_{2}\right)\right) / 10^{m}\right)$. Hence, by Lemma 2.12, $f_{E(x)}(c)=f_{F(y)}(d)$. Thus $f_{E(x)}\left(10^{m}\left(a-x / 10^{m}\right)\right)=$ $f_{E(y)}\left(10^{m}\left(a-y / 10^{m}\right)\right)$.

If $x$ and $y$ differ in more that one coordinate, considering the vectors $\left(x_{1}, y_{2}, \ldots, y_{N}\right),\left(x_{1}, x_{2}, y_{3}, \ldots, y_{N}\right), \ldots,\left(x_{1}, \ldots, x_{N-1}, y_{N}\right)$, we conclude that $h_{m}$ is well defined.

The second part of the lemma follows from Lemma 2.11(b).

\subsection{Lemma. $h$ is well defined and continuous.}

Proof. It is easy to check that $h$ is well defined. From Lemma 2.13 it follows that if $\left(a_{n}, t_{n}\right) \rightarrow(a, t)$ and $t>0$ then $h\left(a_{n}, t_{n}\right) \stackrel{\mathrm{s}}{\rightarrow} h(a, t)$. Thus $h$ is continuous at $(a, t)$ if $t>0$.

Now take a point $(a, 0) \in I^{N+1}$; we will check that $h$ is continuous at this point. Let $\varepsilon>0$. Consider the metric $\mathrm{d}_{0}$ in $I^{N}$ defined by $\mathrm{d}_{0}(b, c)=$ $\max \left\{\left|b_{i}-c_{i}\right|: 1 \leq i \leq N\right\}$. Let $\delta>0$ be such that $\mathrm{d}_{0}(a, b) \leq \delta$ implies that $\mathcal{H}(g(a), g(b))<\varepsilon$. Let $A_{0}=\left[a_{1}-\delta, a_{1}+\delta\right] \times \ldots \times\left[a_{N}-\delta, a_{N}+\delta\right]$ and let $A=\bigcup\left\{g(b): b \in A_{0} \cap I^{N}\right\}$. Then $A$ is a subcontinuum of $X$ and $A \subset N(\varepsilon, g(a))$. Fix $M \in \mathbb{N}$ such that $3 / 10^{M}<\delta$.

We will prove that $h(b, t) \subset N(\varepsilon, h(a, 0))$ for $(b, t) \in I^{N+1}$ such that $\mathrm{d}_{0}(a, b) \leq 1 / 10^{M}$ and $t<1 / 2^{M}$.

Given $m \geq M$, let $x \in\left(\left\{0,1, \ldots, 10^{m}-1\right\}\right)^{N}$ be such that $b \in Q(x)$. If $\sigma \in S_{N}$, then $\mathrm{d}_{0}\left(a,(x+\sigma) / 10^{m}\right)=\max \left\{\left|a_{i}-\left(x_{i}+\sigma_{i}\right) / 10^{m}\right|: 1 \leq i \leq\right.$ $N\} \leq \delta$. Thus $g\left((x+\sigma) / 10^{m}\right) \subset A$ for each $\sigma \in S_{N}$. By Lemma 2.11(c), $f_{E(x)}\left(10^{m}\left(b-x / 10^{m}\right)\right) \subset A$. Therefore $h_{m}(b) \subset A$ for each $m \geq M$. It follows that $h(b, t) \subset A \subset N(\varepsilon, h(a, 0))$.

Now suppose that $h$ is not continuous at $(a, 0)$. Then there exists $B \in$ $\mu^{-1}\left(t_{0}\right)-\{h(a, 0)\}$ and a sequence $\left(\left(a_{n}, t_{n}\right)\right)_{n}$ such that $\left(a_{n}, t_{n}\right) \rightarrow(a, 0)$ and $h\left(a_{n}, t_{n}\right) \rightarrow B$. By the paragraph above, for each $\varepsilon>0$, there exists $K \in \mathbb{N}$ such that $h\left(a_{n}, t_{n}\right) \subset N(\varepsilon, h(a, 0))$ for every $n \geq K$. This implies that $B \subset h(a, 0)$, so $B=h(a, 0)$. This contradiction completes the proof of the continuity of $h$. 
2.16. Lemma. Let $g, g^{*}: I^{N} \rightarrow \mu^{-1}\left(t_{0}\right)$ be maps such that $g \mid \operatorname{Fr}\left(I^{N}\right)=$ $g^{*} \mid \operatorname{Fr}\left(I^{N}\right)$. Let $h, h^{*}: I^{N+1} \rightarrow \mu^{-1}\left(t_{0}\right)$ be the maps constructed as in 2.13 for the maps $g$ and $g^{*}$ respectively. Then $h\left|\operatorname{Fr}\left(I^{N}\right) \times I=h^{*}\right| \operatorname{Fr}\left(I^{N}\right) \times I$ and $h\left|I^{N} \times\{1\}=h^{*}\right| I^{N} \times\{1\}$.

Proof. Consider $h_{m}^{*}, E^{*}(x)$ and $A_{\sigma}^{*}$ constructed as in 2.13 for the map $g^{*}$. Let $(a, t) \in \operatorname{Fr}\left(I^{N}\right) \times I$. If $t=0$, then $h(a, t)=g(a)=g^{*}(a)=h^{*}(a, t)$. Now suppose that $t>0$. To prove that $h(a, t)=h^{*}(a, t)$, it is enough to prove that $h_{m}(a)=h_{m}^{*}(a)$ for every $m \geq 0$. Let $x=\left(x_{1}, \ldots, x_{N}\right) \in$ $\left(\left\{0,1, \ldots, 10^{m}-1\right\}\right)^{N}$ be such that $a \in Q(x)$. We have to prove that $f_{E(x)}\left(10^{m}\left(a-x / 10^{m}\right)\right)=f_{E^{*}(x)}\left(10^{m}\left(a-x / 10^{m}\right)\right)$. Since $a \in \operatorname{Fr}\left(I^{N}\right)$, there exists $r \in\{1, \ldots, N\}$ such that $a_{r}=0$ or 1 .

If $a_{r}=0$, then $x_{r}=0$. We will apply Lemma 2.13 to $p=q=0$. Given $\sigma_{1} \in S_{r-1}$ and $\sigma_{2} \in S_{N-r}, A_{\left(\sigma_{1}, 0, \sigma_{2}\right)}=g\left(\left(x+\left(\sigma_{1}, 0, \sigma_{2}\right)\right) / 10^{m}\right)=g^{*}((x+$ $\left.\left.\left(\sigma_{1}, 0, \sigma_{2}\right)\right) / 10^{m}\right)=A_{\left(\sigma_{1}, 0, \sigma_{2}\right)}^{*}$. Thus Lemma 2.13 implies that $f_{E(x)}\left(10^{m}(a-\right.$ $\left.\left.x / 10^{m}\right)\right)=f_{E^{*}(x)}\left(10^{m}\left(a-x / 10^{m}\right)\right)$.

If $a_{r}=1$, then $x_{r}+1=10^{m}$ and $a_{r}-x_{r} / 10^{m}=1 / 10^{m}$. Set $p=q=1$. Given $\sigma_{1} \in S_{r-1}$ and $\sigma_{2} \in S_{N-r}, A_{\left(\sigma_{1}, 1, \sigma_{2}\right)}=g\left(\left(x+\left(\sigma_{1}, 1, \sigma_{2}\right)\right) / 10^{m}\right)=$ $g^{*}\left(\left(x+\left(\sigma_{1}, 1, \sigma_{2}\right)\right) / 10^{m}\right)=A_{\left(\sigma_{1}, 1, \sigma_{2}\right)}^{*}$. Thus Lemma 2.13 implies that $f_{E(x)}\left(10^{m}\left(a-x / 10^{m}\right)\right)=f_{E^{*}(x)}\left(10^{m}\left(a-x / 10^{m}\right)\right)$. Hence $h(a, t)=$ $h^{*}(a, t)$.

Now take $a \in I^{N}$. We will prove that $h(a, 1)=h^{*}(a, 1)$. Notice that $h(a, 1)=h_{0}(a)=f_{E(0)}(a)$ and $h^{*}(a, 1)=f_{E^{*}(0)}(a)$. Given $\sigma \in S_{N} \subset$ $\operatorname{Fr}\left(I^{N}\right)$, we have $A_{\sigma}=g(\sigma)=g^{*}(\sigma)=A_{\sigma}^{*}$. Thus $f_{E(0)}=f_{E^{*}(0)}$. Therefore $h(a, 1)=h^{*}(a, 1)$.

\subsection{Theorem. Every map $G: S^{N} \rightarrow \mu^{-1}\left(t_{0}\right)$ is null homotopic.}

Proof. Let $G: S^{N} \rightarrow \mu^{-1}\left(t_{0}\right)$ be a map. Let $\left(S^{N}\right)^{+}$and $\left(S^{N}\right)^{-}$be the north and south hemispheres of $S^{N}$ respectively. Let $g=G \mid\left(S^{N}\right)^{+}$ and $g^{*}=G \mid\left(S^{N}\right)^{-}$. Then $g\left|\operatorname{Fr}\left(\left(S^{N}\right)^{+}\right)=g^{*}\right| \operatorname{Fr}\left(\left(S^{N}\right)^{-}\right)$. Identifying $\left(S^{N}\right)^{+}$and $\left(S^{N}\right)^{-}$with $I^{N}$, we consider $h$ and $h^{*}$ as in Lemma 2.16. Then $h\left|\left(\operatorname{Fr}\left(\left(S^{N}\right)^{+}\right) \times I\right) \cup\left(\left(S^{N}\right)^{+} \times\{1\}\right)=h^{*}\right|\left(\operatorname{Fr}\left(\left(S^{N}\right)^{-}\right) \times I\right) \cup\left(\left(S^{N}\right)^{-} \times\{1\}\right)$. We consider the $(N+1)$-ball $B^{N+1}$ as the space obtained by identifying, in the disjoint union $\left(\left(S^{N}\right)^{+} \times I\right) \stackrel{\circ}{\cup}\left(\left(S^{N}\right)^{-} \times I\right)$, the points of the set $\left(\operatorname{Fr}\left(\left(S^{N}\right)^{+}\right) \times I\right) \cup\left(\left(S^{N}\right)^{+} \times\{1\}\right)$ with the points of the set $h^{*} \mid\left(\operatorname{Fr}\left(\left(S^{N}\right)^{-}\right) \times\right.$ $I) \cup\left(\left(S^{N}\right)^{-} \times\{1\}\right)$ in the natural way. Then there exists a map $\bar{h}: B^{N+1} \rightarrow$ $\mu^{-1}\left(t_{0}\right)$ which extends both $h$ and $h^{*}$. Thus $\bar{h}$ is an extension of $G$. Hence $G$ is null homotopic.

Remark. Related with this topic, the following question by A. Petrus ([13]) remains open: If $X$ is a contractible dendroid, is then every Whitney level for $C(X)$ contractible? 


\section{References}

[1] C. Eberhart and S. B. Nadler, Jr., The dimension of certain hyperspaces, Bull. Acad. Polon. Sci. 19 (1971), 1027-1034.

[2] S. Eilenberg, Transformations continues en circonférence et la topologie du plan, Fund. Math. 26 (1936), 61-112.

[3] A. Illanes, Arc-smoothness and contractibility in Whitney levels, Proc. Amer. Math. Soc. 110 (1990), 1069-1074.

[4] - Spaces of Whitney maps, Pacific J. Math. 139 (1989), 67-77.

[5] - The space of Whitney levels, Topology Appl., to appear.

[6] -, Spaces of Whitney decompositions, An. Inst. Mat. Univ. Nac. Autónoma México 28 (1988), 47-61.

[7] - The space of Whitney levels is homeomorphic to $l_{2}$, Colloq. Math., to appear.

[8] -, Arc smoothness is not a Whitney reversible property, Aportaciones Mat.: Comun. 8 (1990), 65-80.

[9] J. Krasinkiewicz and S. B. Nadler, Jr., Whitney properties, Fund. Math. 98 (1978), 165-180.

[10] S. Mardešić, Equivalence of singular and Čech homology for ANR-s. Application to unicoherence, ibid. 46 (1958), 29-45.

[11] S. B. Nadler, Jr., Some basic connectivity properties of Whitney maps inverses in $C(X)$, in: Studies in Topology, Proc. Charlotte Topology Conference (University of North Carolina at Charlotte, 1974), Academic Press, New York 1975, 393-410.

[12] - , Hyperspaces of Sets, Dekker, New York 1978.

[13] A. Petrus, Contractibility of Whitney continua in $C(X)$, Gen. Topology Appl. 9 (1978), 275-288.

[14] L. E. Ward, Jr., Extending Whitney maps, Pacific J. Math. 93 (1981), 465-469.

INSTITUTO DE MATEMÁTICAS

AREA DE LA INVESTIGACIÓN CIENTÍFICA

CIRCUITO EXTERIOR

CIUDAD UNIVERSITARIA

C.P. 04510

MÉXICO, D.F., MÉXICO 